International Journal of Solids and Structures

Editorial manuscript ref. no: IJSS 01-511

\title{
Numerical simulation of debonding of adhesively bonded joint
}

\author{
F. Krasucki ${ }^{a} \quad$ A. Münch ${ }^{b, c} \quad$ Y. Ousset ${ }^{b, 1}$ \\ ${ }^{a}$ Laboratoire de Mécanique et de Génie Civil, UMR 5508 Université Montpellier II, Place Eugène Bataillon, \\ 34695 Montpellier Cedex 5, France \\ ${ }^{b}$ Office National d'Etudes et de Recherches Aérospatiales, BP 72, 92322 Châtillon, France \\ ${ }^{c}$ Laboratoire de Modélisation Mécanique, Université Pierre et Marie Curie, 8 rue du Capitaine Scott, 75015 \\ Paris
}

\begin{abstract}
This paper describes a numerical method to simulate the debonding of adhesively bonded joints. Assuming that the adhesive thickness and the adhesive Young's modulus are small with respect to the characteristic length of the joint and to the Young's modulus of the adherents, a simplified model is derived in the case of large displacements using the asymptotic expansion technique. Then, the problem of the crack growth is stated, in the case of a stable growth, as the search of the local minima of the total energy of the joint, sum of the mechanical energy and the Griffith's fracture energy. This is made using the Newton's method. To this end, the expressions of the first and second derivatives of the mechanical energy with respect to a crack front displacement are derived analytically. Finally, numerical examples are presented, highlighting the unstable character of the crack growth at initiation.
\end{abstract}

Keywords: bonded joint; brittle fracture mechanics; numerical analysis

\section{Introduction}

Both civil and military aircraft structures are made of complex assemblies. For such structures, the main used technique for bonding is the riveting. Unfortunately, when applied to composite materials structural elements, riveting leads to stress concentrations near the holes which are responsible for delamination. Another attractive technique, essentially considered at the moment for repair, is the gluing. If it avoids the piercing of the composite, it does not suppress the zones of stress concentration. Such zones exist near the intersections of the adherent / adhesive interfaces with the free edges. Due

\footnotetext{
${ }^{1}$ Corresponding author. Fax +33-1-4673-4891. Tel +33-1-4673-4663. E-mail address: Yves.Ousset@onera.fr
} 
to the thinness of the adhesive layer, an accurate determination of the stresses in these zones in a global analysis of the structures is irrelevant as it requires a fi ne mesh leading to a great increase of the number of degrees of freedom and ill-conditioning (Geymonat et al. (1998)).

There are two ways to study adhesively bonded joints. The fi rst way consists in developing numerical methods to determine the stress singularities and intensity factors leading to the development of specifi c post-processors (Destuynder et al. (1988)). The second way consists in developing simplifi ed models of the adhesive behaviour. The fi rst approach in this way is based on the analysis of the stress fi eld within the joint. Initiated by the work of Goland and Reissner (1944), it was developed by Wooley and Carver (1971), Delale et al. (1981), Harris and Adams (1984). As the assumption of slender substrates is often made, this approach is devoted to plates assembly. Another approach consists in replacing the adhesive layer by a springs layer. These springs connect the displacements jumps through the adhesive to the stress vector, the constitutive law being either rheological (Rose (1987), Reddy and Roy (1988), Edlund (1994)) or obtained using an asymptotic expansion technique (Klarbring (1991), Destuynder et al. (1992), Geymonat et al. (1999)). In this simplifi ed model, the joint disappears from a geometrical point of view and is replaced by its mid-surface.

In the case of small displacements, a fracture model of the debonding was rigourously developed (Destuynder et al. (1992)) justifying (Bruno and Grimaldi (1990)). It is assumed that the crack was developing in the whole thickness of the joint, the crack front remaining perpendicular to the interfaces. Only the expression of the energy release rate was obtained, the crack growth was not studied. The crack growth was modelled in the case of delamination in composite plates assuming, if the growth is stable, that the front at arrest minimizes the total energy of the plate, sum of the mechanical energy and of the Griffi th's fracture energy (Ousset (1999)). This approach comes into the framework of a revisiting brittle theory developed in (Francfort and Marigo (1998)) and based on minimization of energy.

As the adhesive is assumed much less stiff than the adherents, large displacements will occur in the joint before debonding. As a consequence, the purpose of this paper is to extend the previous approaches in the case of large displacements. The problem will be presented in the paragraph 2 and the simplifi ed model will be developed using the asymptotic expansions technique. The crack growth model will be described in the paragraph 3 and the expressions of the fi rst and second derivatives of the mechanical energy with respect to a crack extension will be derived using the so-called $\theta$ method (Destuynder and Djaoua (1981)). Some comments on the simplifi ed model will be made in the paragraph 4. Then, the numerical approximation and the algorithm are detailed in paragraph 5. At last, some numerical applications will be presented in the paragraph 6 . In this paper, the repeated summation convention is used: Latin $i, j, k, \ldots$ (resp. Greek $\alpha, \beta, \ldots$ ) indices and exponents take their values in the set $\{1,2,3\}$ (resp. $\{1,2\}$ ). 


\section{Problem statement}

\subsection{The 3D elasticity problem}

Let us consider, in the Euclidean space $\mathbb{R}^{3}$ referred to the orthonormal frame $\left(O ; \boldsymbol{e}_{\mathbf{1}}, \boldsymbol{e}_{\mathbf{2}}, \boldsymbol{e}_{\mathbf{3}}\right)$, a bonded assembly (see Fig. 1) $\Omega_{\epsilon}$ formed by two adherents $\Omega_{\epsilon}^{ \pm}$connected by an adhesive layer $\Omega_{\epsilon}^{m}$ of thickness $\epsilon h$ :

$$
\left.\Omega_{\epsilon}=\Omega_{\epsilon}^{ \pm} \cup \Omega_{\epsilon}^{m} \quad ; \quad \Omega_{\epsilon}^{m}=S \times\right]-\frac{\epsilon h}{2}, \frac{\epsilon h}{2}[,
$$

where $\epsilon$ is a small dimensionless parameter, $h$ is a global characteristic length of the structure and $S$ is the mid-surface of $\Omega_{\epsilon}^{m}$. We defi ne the two surfaces $S_{\epsilon}^{+}=\bar{\Omega}_{\epsilon}^{+} \cap \bar{\Omega}_{\epsilon}^{m}$ and $S_{\epsilon}^{-}=\bar{\Omega}_{\epsilon}^{-} \cap \bar{\Omega}_{\epsilon}^{m}$. Besides, the stiffness of the adhesive is assumed to be of order $\epsilon$ compared to the adherent's ones. The structure is fi xed on the part $\Gamma_{u}^{ \pm} \subset \partial \Omega_{\epsilon}^{ \pm}$and is submitted to surface loads $\lambda \boldsymbol{f}^{\epsilon} \in\left(L^{2}\left(\Gamma_{f}^{ \pm}\right)\right)^{3}$ on $\Gamma_{f}^{ \pm} \subset \partial \Omega_{\epsilon}^{ \pm}$ such that $\Gamma_{u}^{ \pm} \cap \Gamma_{f}^{ \pm}=\emptyset ; \lambda$ is the loading factor. It is assumed that a crack is developing on the whole thickness of the adhesive and that the crack front $C_{f}$ remains orthogonal to the mid-surface $S$ during propagation. To simplify the present analysis, we assume that the crack front $C_{f}$ is unloaded.

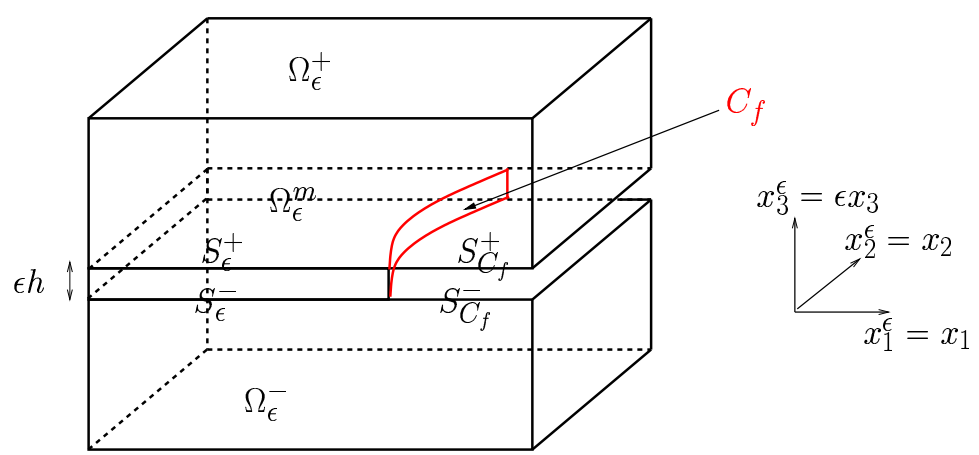

Figure 1: Bonded assembly.

A Lagrangian formulation is used to describe the large displacement motion of the structure and it is assumed that the elastic materials are of the St-Venant Kirchhoff type (Ciarlet (1988)). The constitutive equations are then linear relations between the Green-Lagrange strain tensor $\gamma\left(\boldsymbol{u}^{\epsilon}\right)$ and the second Piola-Kirchhoff stress tensor $\sigma^{\epsilon}$ :

$$
\boldsymbol{\sigma}^{\epsilon}=\mathbb{R}^{\epsilon}: \gamma\left(\boldsymbol{u}^{\epsilon}\right) \quad ; \quad \gamma(\boldsymbol{u})=\frac{1}{2}\left(\boldsymbol{F}^{\boldsymbol{t}} \boldsymbol{F}-\boldsymbol{I}_{\mathbf{3}}\right) \quad ; \quad \boldsymbol{F}=\boldsymbol{I}_{\mathbf{3}}+\nabla \boldsymbol{u}
$$

where $\mathbb{R}^{\epsilon}$ is the fourth order material stiffness tensor and let $\mathbb{S}^{\epsilon}$ be its inverse, $F$ is the gradient of the transformation $\boldsymbol{x} \rightarrow \boldsymbol{x}+\boldsymbol{u}(\boldsymbol{x}), \boldsymbol{I}_{\mathbf{3}}$ is the identity tensor of $\mathbb{R}^{3}$ and $\boldsymbol{\nabla} \boldsymbol{u}=\left(\frac{\partial u_{i}}{\partial x_{j}^{\epsilon}}\right)$. Then, the mechanical 
energy of $\Omega_{\epsilon}$ is written as :

$$
J\left(\boldsymbol{u}^{\boldsymbol{\epsilon}}, \boldsymbol{\sigma}^{\boldsymbol{\epsilon}}, C_{f}\right)=\frac{1}{2} \int_{\Omega_{\epsilon}} \operatorname{Tr}\left(\boldsymbol{\sigma}^{\boldsymbol{\epsilon}} \cdot \boldsymbol{\gamma}\left(\boldsymbol{u}^{\boldsymbol{\epsilon}}\right)\right) d \Omega-\lambda \int_{\Gamma_{f}^{ \pm}} \boldsymbol{f}^{\epsilon} \cdot \boldsymbol{u}^{\boldsymbol{\epsilon}} d \Gamma
$$

where $\operatorname{Tr}$ is the trace operator. The minimum of $J$ with respect to $\boldsymbol{u}^{\epsilon}$ is characterized by the following variational problem :

$$
\left\{\begin{array}{l}
\int_{\Omega_{\epsilon}} \operatorname{Tr}\left(\mathbb{S}^{\epsilon}: \boldsymbol{\sigma}^{\boldsymbol{\epsilon}} \cdot \boldsymbol{\tau}\right) d \Omega_{\epsilon}=\int_{\Omega_{\epsilon}} \operatorname{Tr}\left(\boldsymbol{\tau} \cdot \boldsymbol{\gamma}\left(\boldsymbol{u}^{\epsilon}\right)\right) d \Omega_{\epsilon} \quad \forall \boldsymbol{\tau} \in \Sigma\left(\Omega_{\epsilon}\right) \\
\int_{\Omega_{\epsilon}} \operatorname{Tr}\left(\boldsymbol{\sigma}^{\boldsymbol{\epsilon}} . \boldsymbol{F}^{\boldsymbol{t}} \cdot \boldsymbol{\nabla} \boldsymbol{v}\right) d \Omega_{\epsilon}=\lambda \int_{\Gamma_{f}^{ \pm}} \boldsymbol{f}^{\boldsymbol{\epsilon}} \cdot \boldsymbol{v} d \Gamma \quad \forall \boldsymbol{v} \in V\left(\Omega_{\epsilon}\right)
\end{array}\right.
$$

with $V\left(\Omega_{\epsilon}\right)=\left\{\boldsymbol{v} \in\left(W^{1,4}\left(\Omega_{\epsilon}\right)\right)^{3} / v_{\mid \Gamma_{u}}=0\right\}$ and $\Sigma\left(\Omega_{\epsilon}\right)=\left(L_{s}^{2}\left(\Omega_{\epsilon}\right)\right)^{9}$ (Ciarlet (1988)). We assume that the formulation (4) has at least one solution $\left(\boldsymbol{u}^{\epsilon}, \boldsymbol{\sigma}^{\epsilon}\right)$ in $V\left(\Omega_{\epsilon}\right) \times \Sigma\left(\Omega_{\epsilon}\right)$.

\subsection{The elastic interface model}

To take into account the hypothesis on the stiffness of the constituents, three tensors $\mathbb{R}^{+}, \mathbb{R}^{-}$and $\mathbb{R}^{m}$ are introduced such that $\mathbb{R}^{\epsilon}=\mathbb{R}^{ \pm}$on $\Omega_{\epsilon}^{ \pm}, \mathbb{R}^{\epsilon}=\mathbb{R}^{m}$ on $\Omega_{\epsilon}^{m}$ and $\mathbb{R}^{m} \approx \epsilon \mathbb{R}^{ \pm}$. In order to derive a simplifi ed model taking into account both the material and the geometrical hypothesis, an asymptotic analysis is made, following the method developed in (Ciarlet and Destuynder (1979)). The mechanical energy (3) and the variational formulation (4) are expressed in domains $\Omega^{ \pm}$and $\Omega^{m}$ independent of $\epsilon$ performing a scaling in the direction $x_{3}^{\epsilon}$ in the adhesive by setting $x_{3}^{\epsilon}=\epsilon x_{3}$. The unknowns are written as :

$$
\boldsymbol{u}^{\epsilon}\left(\boldsymbol{x}^{\epsilon}\right)=\boldsymbol{u}(\epsilon, \boldsymbol{x}) \quad ; \quad \boldsymbol{\sigma}^{\epsilon}\left(\boldsymbol{x}^{\epsilon}\right)=\boldsymbol{\sigma}(\epsilon, \boldsymbol{x})
$$

whereas the applied load is scaled as :

$$
\boldsymbol{f}^{\epsilon}\left(\boldsymbol{x}^{\epsilon}\right)=\epsilon \boldsymbol{f}^{\mathbf{1}}(x)
$$

Then, the solution $(\boldsymbol{u}(\epsilon, \boldsymbol{x}), \boldsymbol{\sigma}(\epsilon, \boldsymbol{x}))$ is formally sought under the form of a power expansion of $\epsilon$ :

$$
(\boldsymbol{u}(\epsilon, \boldsymbol{x}), \boldsymbol{\sigma}(\epsilon, \boldsymbol{x}))=\epsilon(\boldsymbol{u}(\boldsymbol{x}), \boldsymbol{\sigma}(\boldsymbol{x}))+\epsilon^{2}\left(\boldsymbol{u}^{\mathbf{2}}(\boldsymbol{x}), \boldsymbol{\sigma}^{\mathbf{2}}(\boldsymbol{x})\right)+o\left(\epsilon^{2}\right)
$$

and the terms of same order in $\epsilon$ are identifi ed. Coming back to the initial variable $\boldsymbol{x}^{\epsilon}$, the leading term of the expansion verifi es the following properties :

- The adherents perform small displacements, so that the strain tensor is reduced to its linear part :

$$
\gamma\left(\boldsymbol{u}^{\epsilon}\right)=\gamma_{\mid l i n}\left(\boldsymbol{u}^{\epsilon}\right) \equiv \frac{1}{2}\left(\left(\nabla \boldsymbol{u}^{\epsilon}\right)^{t}+\nabla \boldsymbol{u}^{\boldsymbol{\epsilon}}\right) \quad \text { in } \quad \Omega_{\epsilon}^{ \pm}
$$


- In the adhesive, the displacements are linear with respect to $x_{3}^{\epsilon}$ :

$$
\boldsymbol{u}^{\epsilon}\left(\boldsymbol{x}^{\epsilon}\right)=\frac{\boldsymbol{u}^{+}\left(x_{\alpha}^{\epsilon}\right)+\boldsymbol{u}^{-}\left(x_{\alpha}^{\epsilon}\right)}{2}+\frac{x_{3}^{\epsilon}}{\epsilon h}\left[\boldsymbol{u}^{\epsilon}\right]\left(x_{\alpha}^{\epsilon}\right) \quad \text { in } \quad \Omega_{\epsilon}^{m},
$$

where $\boldsymbol{u}^{+}\left(x_{\alpha}^{\epsilon}\right)=\boldsymbol{u}\left(x_{\alpha}^{\epsilon}, \frac{\epsilon h}{2}\right), \boldsymbol{u}^{-}\left(x_{\alpha}^{\epsilon}\right)=\boldsymbol{u}\left(x_{\alpha}^{\epsilon},-\frac{\epsilon h}{2}\right)$ and the displacement jump $\left[\boldsymbol{u}^{\epsilon}\right]\left(x_{\alpha}^{\epsilon}\right)=$ $\boldsymbol{u}^{+}\left(x_{\alpha}^{\epsilon}\right)-\boldsymbol{u}^{-}\left(x_{\alpha}^{\epsilon}\right)$ whereas the stresses are independent of $x_{3}^{\epsilon}$ :

$$
\sigma_{i j}^{\epsilon m}=\frac{\mathbb{R}_{i j \alpha 3}^{m}}{\epsilon h}\left[u_{\alpha}^{\epsilon}\right]+\frac{\mathbb{R}_{i j 33}^{m}}{\epsilon h}\left(\left[u_{3}^{\epsilon}\right]+\frac{1}{2 \epsilon h}\left[u_{k}^{\epsilon}\right]\left[u_{k}^{\epsilon}\right]\right) \quad \text { in } \quad \Omega_{\epsilon}^{m} .
$$

- The continuity of the stress vector at the interfaces $S^{ \pm}$leads to the relation :

$$
\sigma_{i 3}^{\epsilon \pm}=\sigma_{i 3}^{\epsilon m}+\frac{\left[u_{i}^{\epsilon}\right]}{\epsilon h} \sigma_{33}^{\epsilon m} \quad \text { on } \quad S^{ \pm}
$$

- To be admissible, the solution $\boldsymbol{u}$ must satisfy the orientation-preserving condition $\operatorname{det} \boldsymbol{F}\left(\boldsymbol{u}^{\boldsymbol{\epsilon}}\right)>$ 0 in $\Omega_{\epsilon}$ (Ciarlet (1988)). This condition is, at the fi rst order, automatically verifi ed in the adherents where $\operatorname{det} \boldsymbol{F}\left(\boldsymbol{u}^{\epsilon}\right)=1+O(\epsilon)$, whereas in the adhesive, $\operatorname{det} \boldsymbol{F}\left(\boldsymbol{u}^{\epsilon}\right)=\left[u_{3}^{\epsilon}\right]+\epsilon h+O\left(\epsilon^{2}\right)$ prescribes the condition :

$$
\left[u_{3}^{\epsilon}\right]>-\epsilon h .
$$

Finally, the mechanical energy associated to the simplifi ed model is :

$$
\begin{aligned}
J\left(\boldsymbol{u}^{\epsilon}, \boldsymbol{\sigma}^{\boldsymbol{\epsilon}}, c_{f}\right)=\frac{1}{2} & \int_{\Omega_{\epsilon}^{ \pm}} \operatorname{Tr}\left(\boldsymbol{\sigma}^{\epsilon} \cdot \boldsymbol{\gamma}_{\mid \boldsymbol{i n n}}\left(\boldsymbol{u}^{\boldsymbol{\epsilon}}\right)\right) d \Omega \\
& -\frac{1}{2} \int_{S}\left[\sigma_{\alpha 3}^{\epsilon}\left[u_{\alpha}^{\epsilon}\right]+\sigma_{33}^{\epsilon}\left(\left[u_{3}^{\epsilon}\right]+\frac{1}{2 \epsilon h}\left[u_{k}^{\epsilon}\right]\left[u_{k}^{\epsilon}\right]\right)\right] d S-\lambda \int_{\Gamma_{f}^{ \pm}} \boldsymbol{f}^{\epsilon} \cdot \boldsymbol{u}^{\boldsymbol{\epsilon}} d \Gamma
\end{aligned}
$$

where the term over $\Omega_{\epsilon}^{m}$ has been integrated in the thickness. This functional admits non unique local minima (Krasucki et al. (2001)) in the functional space $\tilde{V}\left(\Omega_{\epsilon}^{ \pm}\right)=\left\{\boldsymbol{v}^{ \pm} \in\left(H^{1}\left(\Omega_{\epsilon}^{ \pm}\right)\right)^{3}, \boldsymbol{v}^{ \pm}=\right.$ 0 on $\left.\Gamma_{u}^{ \pm}\right\}$, characterized by the following variational equation :

$$
\int_{\Omega_{\epsilon}^{ \pm}} \operatorname{Tr}\left(\boldsymbol{\sigma}^{\epsilon} \cdot \boldsymbol{\nabla} \boldsymbol{v}\right) d \Omega+\int_{S}\left[\sigma_{\alpha 3}^{\epsilon}\left[v_{\alpha}\right]+\sigma_{33}^{\epsilon}\left(\left[v_{3}\right]+\frac{1}{\epsilon h}\left[u_{k}^{\epsilon}\right]\left[v_{k}\right]\right)\right] d S=\lambda \int_{\Gamma_{f}^{ \pm}} \boldsymbol{f}^{\epsilon} \cdot \boldsymbol{v} d \Gamma \quad \forall \boldsymbol{v} \in \tilde{V}\left(\Omega_{\epsilon}^{ \pm}\right) .
$$

As in the linear case (Klarbring (1991), Destuynder et al. (1992), Geymonat et al. (1999)), in the simplifi ed model the joint disappears from a geometrical point of view and is replaced by an energy of adhesion defi ned on its mid-surface. Finally, the tangent stiffness operator of problem (12) is :

$$
\begin{gathered}
T\left(\boldsymbol{u}^{\epsilon}, \boldsymbol{v}\right) \cdot \boldsymbol{w}=\int_{\Omega_{\epsilon}^{ \pm}} \operatorname{Tr}\left(\mathbb{R}^{ \pm}: \boldsymbol{\nabla} \boldsymbol{w} \cdot \boldsymbol{\nabla} \boldsymbol{v}\right) d \Omega+\int_{S} \sigma_{\alpha 3}^{\epsilon}(\boldsymbol{v})\left[w_{\alpha}\right] d S+\int_{S} \frac{1}{\epsilon h} \sigma_{33}\left(\boldsymbol{u}^{\epsilon}\right)\left[w_{k}\right]\left[v_{k}\right] d S \\
+\frac{1}{\epsilon h} \int_{S} R_{3333}^{m}\left(\left[w_{3}\right]+\frac{1}{\epsilon h}\left[w_{l}\right]\left[u_{l}^{\epsilon}\right]\right)\left(\left[v_{3}\right]+\frac{1}{\epsilon h}\left[u_{k}^{\epsilon}\right]\left[v_{k}\right]\right) d S
\end{gathered}
$$




\section{The debonding model}

To simplify the expression, we note in the following, $\boldsymbol{u}$ for $\boldsymbol{u}^{\epsilon}$ and $\boldsymbol{\sigma}$ for $\boldsymbol{\sigma}^{\boldsymbol{\epsilon}}$.

\subsection{Overview of the crack growth model}

As the elastic interface model reduces the adhesive domains to its mid-surface $S$, the crack front $C_{f}$ is described by the line $c_{f}=C_{f} \cap S$ (see fi gure 3). The propagation of $c_{f}$, assumed quasi-static, is taken into account by introducing a total energy $E\left(\boldsymbol{u}, c_{f}\right)$ sum of the mechanical energy $J\left(\boldsymbol{u}, c_{f}\right)$ defi ned by the relation (11) and the Griffi th's fracture energy $D\left(c_{f}\right)$ :

$$
E\left(\boldsymbol{u}, c_{f}\right)=J\left(\boldsymbol{u}, c_{f}\right)+D\left(c_{f}\right)
$$

The fracture energy is proportional to the delaminated area :

$$
D\left(c_{f}\right)=G_{c} \int_{S_{c_{f}}} d S
$$

where $G_{c}$ is the critical energy release rate assumed constant along $c_{f}$ and $S_{c_{f}}$ the delaminated part of $S$. Then, the model of propagation is stated as follow: let $c_{f}$ be the crack front at starting and let the loading factor $\lambda$ be unchanged during the growth, it is assumed that the unknown front at arrest and the displacement fi eld $\boldsymbol{u}$ ensure the stationarity of $E\left(\boldsymbol{u}, c_{f}\right)$ seen as a function of two variables :

$$
\text { find }\left(\boldsymbol{u}, c_{f}\right) \text { such that } E\left(\boldsymbol{u}, c_{f}\right)=\min _{(\boldsymbol{v}, c)} E(\boldsymbol{v}, c) \quad \forall \boldsymbol{v} \in V, \forall c \in C,
$$

where $C$ is the set of the admissible front's positions. In order to write the stationarity of $E$ with respect to $c_{f}$, the virtual kinematics of the delamination front is described by a plane vector fi eld $\boldsymbol{\theta}(\boldsymbol{x})=\left(\theta_{1}(\boldsymbol{x}), \theta_{2}(\boldsymbol{x}), 0\right)$ where the components $\theta_{\alpha}$ are regular functions defi ned in $\Omega$ with support $S_{\theta}$ restricted to a small neighbourhood of $c_{f}$. The assumption that $C_{f}$ remains perpendicular to the interfaces during growth dictates the condition $[\boldsymbol{\theta}]=0$ on $S$.

If the front $c_{f}$ intersects the boundary of $S, \boldsymbol{\theta}$ must satisfy in addition the kinematical condition $\boldsymbol{\theta} . \boldsymbol{\nu}=0$ on $S_{\theta} \cap \partial S$ where $\boldsymbol{\nu}$ is the unit normal. Then, in order to obtain the derivatives of $E$ with respect to the front in the direction $\boldsymbol{\theta}$, it is used the so-called $\theta$-method (Destuynder and Djaoua (1981), Ousset (1999)): let $\eta$ be a dimensionless small parameter and a family of mappings associated to the fi eld $\boldsymbol{\theta}$ :

$$
\mathcal{F}^{\eta}: \boldsymbol{x} \in \Omega_{\epsilon} \rightarrow \boldsymbol{x}^{\boldsymbol{\eta}} \in \Omega_{\epsilon}^{\eta} \quad ; \quad \boldsymbol{x}^{\boldsymbol{\eta}}=\boldsymbol{x}+\eta \boldsymbol{\theta}(\boldsymbol{x})
$$

Let $(\boldsymbol{u}, \boldsymbol{\sigma})$ and $\left(\boldsymbol{u}^{\boldsymbol{\eta}}, \boldsymbol{\sigma}^{\boldsymbol{\eta}}\right)$ be the solution of problem (12) set in $\Omega$ and $\Omega^{\eta}$ respectively for the same load. The fi rst and second derivatives of the energy $E$ defi ned as :

$$
E^{(1)}(\boldsymbol{\theta})=\lim _{\eta \rightarrow 0} \frac{E^{\eta}-E}{\eta} \quad, \quad E^{(2)}(\boldsymbol{\theta}, \boldsymbol{\theta})=\lim _{\eta \rightarrow 0} \frac{E^{\eta}-E-\eta E^{(1)} \boldsymbol{\theta}}{\eta^{2}}
$$




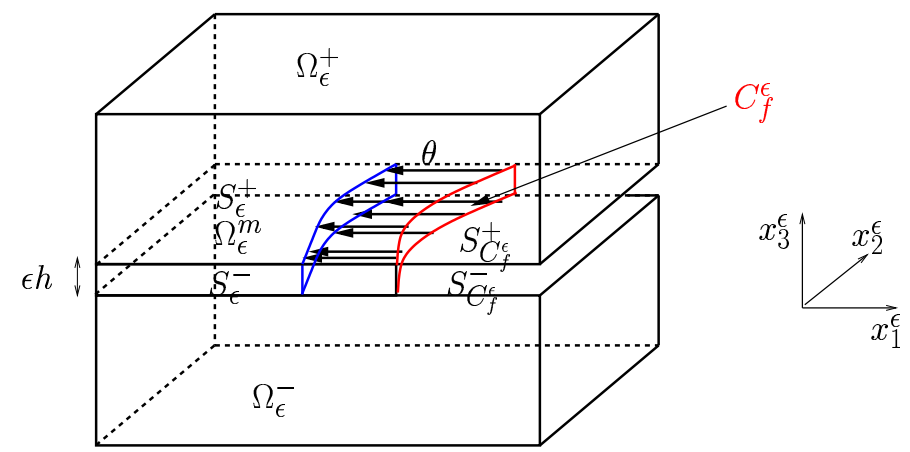

Figure 2: Virtual propagation of $C_{f}$.

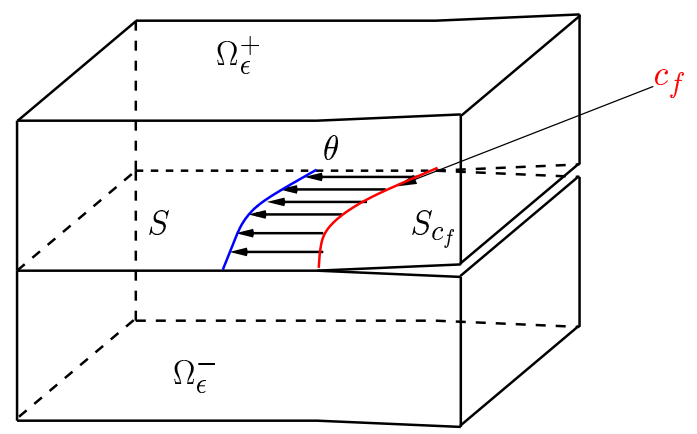

Figure 3: Virtual propagation of $c_{f}$.

are obtained in the following way: the integrals and derivatives over $\Omega_{\epsilon}^{\eta}$ are expressed as integrals and derivatives over $\Omega_{\epsilon}$ using the two following relations :

$$
\begin{aligned}
\boldsymbol{d x}^{\boldsymbol{\eta}} & =\operatorname{det}\left(\boldsymbol{I}_{\mathbf{3}}+\eta \nabla \boldsymbol{\theta}\right) \boldsymbol{d} \boldsymbol{x}=\left(1+\eta \operatorname{div} \boldsymbol{\theta}+\eta^{2} \operatorname{det}(\nabla \boldsymbol{\theta})\right) \boldsymbol{d} \boldsymbol{x} \\
\frac{\partial}{\partial \boldsymbol{x}^{\boldsymbol{\eta}}} & =\frac{\partial}{\partial \boldsymbol{x}} \cdot\left(\boldsymbol{I}_{\mathbf{3}}+\eta \nabla \boldsymbol{\theta}\right)^{-1}=\frac{\partial}{\partial \boldsymbol{x}} \cdot\left(\boldsymbol{I}_{\mathbf{3}}-\eta \nabla \boldsymbol{\theta}+\eta^{2} \nabla \boldsymbol{\theta} . \nabla \boldsymbol{\theta}\right)+o\left(\eta^{2}\right),
\end{aligned}
$$

making clear the $\eta$ dependence, then the solution is expanded as a formal power series of $\eta:\left(\boldsymbol{u}^{\boldsymbol{\eta}}, \boldsymbol{\sigma}^{\boldsymbol{\eta}}\right)=$ $(\boldsymbol{u}, \boldsymbol{\sigma})+\eta\left(\boldsymbol{u}^{\mathbf{1}}, \boldsymbol{\sigma}^{\mathbf{1}}\right)+\eta^{2}\left(\boldsymbol{u}^{\mathbf{2}}, \boldsymbol{\sigma}^{\mathbf{2}}\right)+o\left(\eta^{2}\right)$ and terms of same order are identifi ed. The results of the computation concerning the simplifi ed model, are given in the next sections.

\subsection{First derivatives of the displacements and the stresses}

The fi rst order Lagrangian derivative $\left(\boldsymbol{u}^{\mathbf{1}}, \boldsymbol{\sigma}^{\mathbf{1}}\right)$ are needed to compute the second derivative of $J$; they are the solution of the following variational problem

$$
\left\{\begin{aligned}
\boldsymbol{\sigma}^{\mathbf{1}}= & \mathbb{R}^{ \pm}:\left(\boldsymbol{\nabla} \boldsymbol{u}^{\mathbf{1}}-\boldsymbol{\nabla} \boldsymbol{u} \cdot \boldsymbol{\nabla} \boldsymbol{\theta}\right) \quad \text { on } \Omega_{\epsilon}^{ \pm} \\
\sigma_{\alpha 3}^{1}= & \frac{R_{\alpha 3 \alpha 3}^{m}}{\epsilon h}\left[u_{\alpha}^{1}\right] \quad ; \quad \sigma_{33}^{1}=\frac{R_{3333}^{m}}{\epsilon h}\left(\left[u_{3}^{1}\right]+\frac{1}{\epsilon h}\left[u_{k}^{1}\right]\left[u_{k}\right]\right) \quad \text { on } S ; \\
\int_{\Omega_{\epsilon}^{ \pm}} & \operatorname{Tr}\left(\boldsymbol{\sigma}^{\mathbf{1}} \cdot \boldsymbol{\nabla} \boldsymbol{v}\right) d \Omega+\int_{S}\left[\sigma_{\alpha 3}^{1}\left[v_{\alpha}\right]+\sigma_{33}^{1}\left(\left[v_{3}\right]+\frac{1}{\epsilon h}\left[u_{l}\right]\left[v_{l}\right]\right)+\frac{1}{\epsilon h} \sigma_{33}\left[u_{l}^{1}\right]\left[v_{l}\right]\right] d S \\
& =\int_{\Omega_{\epsilon}^{ \pm}} \operatorname{Tr}(\boldsymbol{\sigma}(\boldsymbol{u}) \cdot \boldsymbol{\nabla} \boldsymbol{v}) \operatorname{div} \boldsymbol{\theta} d \Omega+\int_{\Omega_{\epsilon}^{ \pm}} \operatorname{Tr}(\boldsymbol{\sigma}(\boldsymbol{v}) \cdot \boldsymbol{\nabla} \boldsymbol{u} \cdot \boldsymbol{\nabla} \boldsymbol{\theta}) d \Omega \\
& -\int_{S}\left[\sigma_{\alpha 3}\left[v_{\alpha}\right]+\sigma_{33}\left(\left[v_{3}\right]+\frac{1}{\epsilon h}\left[v_{l}\right]\left[u_{l}\right]\right)\right] \operatorname{div} \boldsymbol{\theta} d S \quad \forall \boldsymbol{v} \in \tilde{V}\left(\Omega^{ \pm}\right) .
\end{aligned}\right.
$$

This equation shows that the Lagrangian derivatives are solution of a linear problem involving the tangent stiffness operator $T(\boldsymbol{u}, \boldsymbol{v}) . \boldsymbol{u}^{\mathbf{1}}$ of problem (4), whereas in the loading term (the right-hand side of the relation), $\boldsymbol{\theta}$ acts as a parameter. 


\subsection{First and second derivatives of the total energy}

Let us note $J^{(1)}(\boldsymbol{\theta})$ the first derivative of the mechanical energy and $g(\boldsymbol{\theta}) \equiv-J^{(1)}(\boldsymbol{\theta})$ the energy release rate. After classical computation, $g$ is expressed as a function of the displacement $\boldsymbol{u}$ and the stress $\boldsymbol{\sigma}$ only. We obtain :

$$
\begin{aligned}
g(\boldsymbol{\theta})= & \int_{\Omega_{\epsilon}^{ \pm}} \operatorname{Tr}(\boldsymbol{\sigma} \cdot \boldsymbol{\nabla} \boldsymbol{u} \cdot \boldsymbol{\nabla} \boldsymbol{\theta}) d \Omega-\frac{1}{2} \int_{\Omega_{ \pm}} \operatorname{Tr}(\boldsymbol{\sigma} \cdot \boldsymbol{\nabla} \boldsymbol{u}) \operatorname{div} \boldsymbol{\theta} d \Omega \\
& -\frac{1}{2} \int_{S}\left[\sigma_{\alpha 3}\left[u_{\alpha}\right]+\sigma_{33}\left(\left[u_{3}\right]+\frac{1}{2 \epsilon h}\left[u_{k}\right]\left[u_{k}\right]\right)\right] \operatorname{div} \boldsymbol{\theta} d S .
\end{aligned}
$$

Integrating by parts and using both the constitutive (7) and the local equilibrium equations derived from the equations (12), it is found (see Appendix B) that the energy release rate can be written as a curvilinear integral along the front $c_{f}$, as follows :

$$
g(\boldsymbol{\theta})=\frac{1}{2} \int_{c_{f}}\left[\sigma_{\alpha 3}\left[u_{\alpha}\right]+\sigma_{33}\left(\left[u_{3}\right]+\frac{1}{2 \epsilon h}\left[u_{l}\right]\left[u_{l}\right]\right)\right] \boldsymbol{\theta} . \boldsymbol{\nu} d \gamma \equiv \int_{c_{f}} G \boldsymbol{\theta} . \boldsymbol{\nu} d \gamma
$$

The integrand $G$ is the so-called delamination force or local energy release rate and can be viewed as the dual variable of $\boldsymbol{\theta} . \boldsymbol{\nu}$ on $c_{f}$. For numerical computations, expression (21) is used as it is less mesh sensitive than expression (22). It gives accurate results with relatively coarse fi nite elements meshes. Let us remark that the quantity $G$, which is a function of the displacement jump across $S$,

$$
G=\frac{1}{2}\left(\sigma_{\alpha 3}\left[u_{\alpha}\right]+\sigma_{33}\left(\left[u_{3}\right]+\frac{1}{2 \epsilon h}\left[u_{l}\right]\left[u_{l}\right]\right)\right)=\frac{1}{2 \epsilon h}\left(R_{\alpha 3 \alpha 3}^{m}\left[u_{\alpha}\right]^{2}+R_{3333}^{m}\left(\left[u_{3}\right]+\frac{1}{2 \epsilon h}\left[u_{l}\right]\left[u_{l}\right]\right)^{2}\right)
$$

is strictly positive, even if a crack doesn't exist.

Finally, the second derivative takes the following form :

$$
\begin{aligned}
J^{(2)}(\boldsymbol{\theta}, \boldsymbol{\theta})= & -\frac{1}{2} \int_{\Omega_{\epsilon}^{ \pm}} \operatorname{Tr}\left(\boldsymbol{\sigma} \cdot \boldsymbol{\nabla} \boldsymbol{u}^{\mathbf{1}} \cdot \boldsymbol{\nabla} \boldsymbol{\theta}\right) d \Omega-\frac{1}{2} \int_{\Omega_{\epsilon}^{ \pm}} \operatorname{Tr}\left(\boldsymbol{\sigma}^{\mathbf{1}} \cdot \boldsymbol{\nabla} \boldsymbol{u} \cdot \boldsymbol{\nabla} \boldsymbol{\theta}\right) d \Omega \\
& +\frac{1}{2} \int_{\Omega_{\epsilon}^{ \pm}} \operatorname{Tr}\left(\boldsymbol{\sigma}^{\mathbf{1}} \cdot \boldsymbol{\nabla} \boldsymbol{u}\right) \operatorname{div} \boldsymbol{\theta} d \Omega+\int_{\Omega_{\epsilon}^{ \pm}} \operatorname{Tr}(\boldsymbol{\sigma} \cdot \boldsymbol{\nabla} \boldsymbol{u} \cdot \boldsymbol{\nabla} \boldsymbol{\theta} \cdot \boldsymbol{\nabla} \boldsymbol{\theta}) d \Omega \\
& -\frac{1}{2} \int_{\Omega_{\epsilon}^{ \pm}} \operatorname{Tr}(\boldsymbol{\sigma} \cdot \boldsymbol{\nabla} \boldsymbol{u} \cdot \boldsymbol{\nabla} \boldsymbol{\theta}) \operatorname{div} \boldsymbol{\theta} d \Omega+\frac{1}{2} \int_{\Omega_{\epsilon}^{ \pm}} \operatorname{Tr}(\boldsymbol{\sigma} \cdot \boldsymbol{\nabla} \boldsymbol{u}) \operatorname{det}(\boldsymbol{\nabla} \boldsymbol{\theta}) d \Omega \\
& +\frac{1}{2} \int_{S}\left[\sigma_{\alpha 3}^{1}\left[u_{\alpha}\right]+\sigma_{33}^{1}\left(\left[u_{3}\right]+\frac{1}{2 \epsilon h}\left[u_{l}\right]\left[u_{l}\right]\right)\right] \operatorname{div} \boldsymbol{\theta} d S \\
& +\frac{1}{2} \int_{S}\left[\sigma_{\alpha 3}\left[u_{\alpha}\right]+\sigma_{33}\left(\left[u_{3}\right]+\frac{1}{2 \epsilon h}\left[u_{l}\right]\left[u_{l}\right]\right)\right] \operatorname{det}(\boldsymbol{\nabla} \boldsymbol{\theta}) d S,
\end{aligned}
$$


where det stands for the determinant $\left(\operatorname{det}(\boldsymbol{\nabla} \boldsymbol{\theta})=\theta_{1,1} \theta_{2,2}-\theta_{1,2} \theta_{2,1}\right) . J^{(2)}$ is a quadratic form associated to a symmetric bilinear form that depends on the solution $(\boldsymbol{u}, \boldsymbol{\sigma})$ and on the first Lagrangian derivatives $\left(\boldsymbol{u}^{\mathbf{1}}, \boldsymbol{\sigma}^{\mathbf{1}}\right)$.

In view of the defi nition of $D$, the derivation of the expansion for the fracture energy derivatives is straightforward :

$$
D^{(1)}(\boldsymbol{\theta})=-G_{c} \int_{S_{c_{f}}} \operatorname{div} \boldsymbol{\theta} d S=G_{c} \int_{c_{f}} \boldsymbol{\theta} . \boldsymbol{\nu} d \gamma \quad ; \quad D^{(2)}(\boldsymbol{\theta}, \boldsymbol{\theta})=-G_{c} \int_{S_{c_{f}}} \operatorname{det}(\boldsymbol{\nabla} \boldsymbol{\theta}) d S .
$$

\subsection{Conditions on $\theta$}

The front $c_{f}$ cannot move back; this requirement is satisfi ed if and only if :

$$
\boldsymbol{\theta} . \boldsymbol{\nu} \geq 0
$$

where $\boldsymbol{\nu}$ is the unit normal to front in the direction of delamination growth. Due to this irreversibility condition, the problem to be solved is a constrained minimization one and can be solved using a fi xed point algorithm. On the other hand, the mapping introduced in section (3.1) must be defi ned in such a way that the delaminated area increases. This requirement takes the following form :

$$
\int_{S_{c_{f}}} d S-\int_{S_{c_{f}}^{\eta}} d S^{\eta}=-\eta \int_{S_{c_{f}}} \operatorname{div} \boldsymbol{\theta} d S-\eta^{2} \int_{S_{c_{f}}} \operatorname{det}(\boldsymbol{\nabla} \boldsymbol{\theta}) d S \geq 0 \quad \forall \eta>0 .
$$

A suffi cient condition for $\boldsymbol{\theta}$ is then $\int_{{ }^{c} S_{d}} \operatorname{div} \boldsymbol{\theta} d S \leq 0$ and $\int_{{ }^{c} S_{d}} \operatorname{det}(\boldsymbol{\nabla} \boldsymbol{\theta}) d S \leq 0$ which is equivalent, in the case of constant $G_{c}$ and according to the relations (25), to $D^{(1)}(\boldsymbol{\theta}) \geq 0$ and $D^{(2)}(\boldsymbol{\theta}, \boldsymbol{\theta}) \geq 0$. The fi rst inequality is satisfi ed as soon as condition (26) is satisfi ed. The set $ઇ$ of admissible displacement fi elds of the front is so :

$$
V_{\theta}=\left\{\boldsymbol{\theta} \in\left(H^{1}\left(S_{\theta}\right)\right)^{2}, \boldsymbol{\theta} . \boldsymbol{\nu} \geq 0 \text { on } c_{f}, \boldsymbol{\theta} . \boldsymbol{\nu}=0 \text { on } S_{\theta} \cap \partial S, D^{(2)}(\boldsymbol{\theta}, \boldsymbol{\theta}) \geq 0\right\} .
$$

The spectrum of the operator $D^{(2)}$ is real and symmetric with respect to zero (Münch and Ousset (2002) or Appendix A). As a consequence, the sets $\left\{\boldsymbol{\theta} ; D^{(2)}(\boldsymbol{\theta}, \boldsymbol{\theta})>0\right\}$ and $V_{\theta}$ are not empty.

Let us now consider stable debonding. According to Nguyen (2000), the debonding is stable, for a fi xed level of loading, if $f^{(2)}(\boldsymbol{\theta}, \boldsymbol{\theta})>0 \forall \boldsymbol{\theta} \in V_{\boldsymbol{\theta}}$. This condition ensures that, in the vicinity of a stable crack front location at arrest, the total energy $E$ is strictly convex : $E^{(2)}(\boldsymbol{\theta}, \boldsymbol{\theta})=J^{(2)}(\boldsymbol{\theta}, \boldsymbol{\theta})+$ $D^{(2)}(\boldsymbol{\theta}, \boldsymbol{\theta})>0$. As a consequence, the growth arrest corresponds to a local minimum of $E$. According to relations (22) and $\left(25_{1}\right)$ this local minimum $\boldsymbol{\theta} \in V_{\theta}$ is characterized by the following variational inequality :

$$
E^{(1)}(\hat{\boldsymbol{\theta}}-\boldsymbol{\theta})=\int_{c_{f}}\left(G_{c}-G\right)(\hat{\boldsymbol{\theta}}-\boldsymbol{\theta}) . \boldsymbol{\nu} d \Gamma \geq 0 \quad \forall \hat{\boldsymbol{\theta}} \in V_{\boldsymbol{\theta}}
$$


This equation is nothing but the variational form of the well-known Griffi th's criterion; it implies $G=G_{c}$ along the front only if $G$ and $c_{f}$ are smooth enough. Inequation (28) is non linear with respect to the variable $\boldsymbol{\theta}$ and can be solve introducing a Lagrangian $L(\boldsymbol{\theta}, \boldsymbol{\mu})=E^{(1)}(\boldsymbol{\theta})+(\boldsymbol{\mu}, \alpha(\boldsymbol{\theta}))$, where $\alpha(\boldsymbol{\theta})=-\boldsymbol{\theta} . \boldsymbol{\nu}$ and $\mu$ the function dual of $\alpha$ and using an interior point technique (Herskovits (1992)). In fact, when the growth is stable, the situation $\boldsymbol{\theta} . \boldsymbol{\nu}<0$ never occurs. The minimum $\boldsymbol{\theta}$ is then characterized by the simpler nonlinear relation $E^{(1)}(\boldsymbol{\theta})=0$ solved using a Newton's method: the front displacement $\Theta \in V_{\theta}$ is computed by solving the following variational problem :

$$
E^{(2)}(\boldsymbol{\Theta}, \boldsymbol{\theta})=-E^{(1)}(\boldsymbol{\theta}) \quad \forall \boldsymbol{\theta} \in V_{\boldsymbol{\theta}}
$$

Let us insist on the point that $E^{(2)}$ is a non local operator. Consequently, the points of the front are not moved independently. The displacement $\Theta$ is proportional to the value $G-G_{c}$ not locally on each point of $c_{f}$ but in a variational sense : if $s \in[0, l]$ designs the curvilinear abscissa along the front $c_{f}$, then the quantity $\Theta(\tau)$ (for any $\tau$ fi xed in $[0, l]$ ) depends non only to $G(\tau)-G_{c}$ but also to $G(s)-G_{c}$ for all $s \in[0, l]$.

\section{Comments on the simplified model}

\subsection{Convergence of the elastic interface model toward the perfect interface model}

The elastic interface model assumes that both the joint thickness and the adhesive Young's modulus tend to zero simultaneously as $\epsilon$. This assumption can shock the physical sense as the Young's modulus is a fi xed data. One can wonder what the model becomes if the joint thickness only tends to zero. This question is answered here in the case of small displacements. In this case, the joint strain energy appears clearly as a penalization of the perfectly bonded interface model condition $\left[\boldsymbol{u}^{\epsilon}\right]=0$ on $S$.

The linear elastic interface model is stated as follows: fi nd $\boldsymbol{u}^{\epsilon} \in W$ such that :

$$
\left\{\begin{array}{l}
\int_{\Omega_{\epsilon}^{ \pm}} \operatorname{Tr}\left(\boldsymbol{\sigma}^{\boldsymbol{\epsilon}} \cdot \boldsymbol{\nabla} \boldsymbol{v}\right) d \Omega^{ \pm}+\frac{1}{\epsilon} \int_{S} R_{i 3 i 3}^{m}\left[u_{i}^{\epsilon}\right]\left[v_{i}\right] d S=\int_{\Gamma_{f}^{ \pm}} \boldsymbol{f}^{\epsilon} \cdot \boldsymbol{v} d \Gamma \quad \forall \boldsymbol{v} \in W \\
\boldsymbol{\sigma}^{\epsilon}=\mathbb{R}^{ \pm}: \nabla \boldsymbol{u}^{\epsilon} \quad \in \Omega_{\epsilon}^{ \pm}
\end{array}\right.
$$

where $W$ is the space $W=\left\{\boldsymbol{v} \mid v_{\mid \Omega_{\epsilon}^{ \pm}} \in\left(H^{1}\left(\Omega^{ \pm}\right)\right)^{3}, \boldsymbol{v}=0\right.$ on $\left.\Gamma_{u}\right\}$ equipped with the norm $\|v\|_{W}$ such that :

$$
\|\boldsymbol{v}\|_{W}^{2}=\int_{\Omega_{\epsilon}^{ \pm}} \operatorname{Tr}(\boldsymbol{\sigma}(\boldsymbol{v}) \cdot \boldsymbol{\nabla} \boldsymbol{v}) d \Omega^{ \pm}+\int_{S} R_{i 3 i 3}^{m}\left[v_{i}\right]^{2} d S
$$

for which $W$ is an Hilbert space (the Korn's inequalities ensure that (31) is a norm equivalent to the classical norm). Then, the solution of the problem (30) is formally sought under the form of a power expansion of $\epsilon$ : $\left(\boldsymbol{\sigma}^{\boldsymbol{\epsilon}}, \boldsymbol{u}^{\boldsymbol{\epsilon}}\right)=\left(\boldsymbol{\sigma}^{\mathbf{0}}, \boldsymbol{u}^{\mathbf{0}}\right)+\epsilon\left(\boldsymbol{\sigma}^{\mathbf{1}}, \boldsymbol{u}^{\mathbf{1}}\right)+o(\epsilon)$. Transferring this expansion into the 
variational equation of (30) and identifying the terms of the same order in $\epsilon$, one obtains that $\left(\boldsymbol{\sigma}^{\mathbf{0}}, \boldsymbol{u}^{\mathbf{0}}\right)$ satisfi es the following equations :

$$
\left\{\begin{array}{l}
\int_{\Omega_{\epsilon}^{ \pm}} \operatorname{Tr}\left(\boldsymbol{\sigma}^{\mathbf{0}} \cdot \boldsymbol{\nabla} \boldsymbol{v}\right) d \Omega=\int_{\Gamma_{f}^{ \pm}} \boldsymbol{f}^{\boldsymbol{\epsilon}} \cdot \boldsymbol{v} d \Gamma \quad \forall \boldsymbol{v} \in V \\
\boldsymbol{\sigma}^{\mathbf{0}}=\mathbb{R}^{ \pm}: \boldsymbol{\nabla} \boldsymbol{u}^{\mathbf{0}} \in \Omega_{\epsilon}^{ \pm}
\end{array}\right.
$$

with $V=\left\{\boldsymbol{v} \in W \mid \boldsymbol{v} \in\left(H^{1}(\Omega)\right)^{3}, \Omega=\Omega_{\epsilon}^{ \pm} \cup S\right\}$. The limit model is nothing but the perfectly bonded interface model where $\left[\boldsymbol{u}^{0}\right]=0$ on $S$. Then, the formal expansion is justifi ed with the

Theorem 1 As $\epsilon$ tends to zero, we have the following convergence results:

- $\left[\boldsymbol{u}^{\epsilon}\right] \rightarrow 0$ in $\left(\mathbb{L}^{2}(S)\right)^{3}$ as $\epsilon$,

- $\boldsymbol{u}^{\epsilon} \rightarrow \boldsymbol{u}^{0}$ in $W$,

- $\boldsymbol{\sigma}^{\boldsymbol{\epsilon}} \rightarrow \boldsymbol{\sigma}^{\mathbf{0}}$ in $\left(\mathbb{L}_{S}^{2}(\Omega)\right)^{9}$,

- $g^{\epsilon}(\boldsymbol{\theta}) \rightarrow g^{0}(\boldsymbol{\theta})$ in $\mathbb{R}$.

Proof: The proof of the fi rst three points follows the approach presented by Lions (1973), Chapter I and is detailed, for instance, in (Ait Moussa (1996)). Now, as $\boldsymbol{\theta}$ is a regular vector fi eld, one can take the limit of the energy release rate expression

$$
g^{\epsilon}(\boldsymbol{\theta})=-\frac{1}{2} \int_{\Omega_{\epsilon}^{ \pm}} \operatorname{Tr}\left(\boldsymbol{\sigma}^{\epsilon} \cdot \boldsymbol{\nabla} \boldsymbol{u}^{\boldsymbol{\epsilon}}\right) d i v \boldsymbol{\theta} d \Omega+\int_{\Omega_{\epsilon}^{ \pm}} \operatorname{Tr}\left(\boldsymbol{\sigma}^{\boldsymbol{\epsilon}} \cdot \boldsymbol{\nabla} \boldsymbol{u}^{\epsilon} \cdot \boldsymbol{\nabla} \boldsymbol{\theta}\right) d \Omega-\frac{1}{2 \epsilon} \int_{S} R_{i 3 i 3}^{m}\left[u_{i}^{\epsilon}\right]^{2} d i v \boldsymbol{\theta} d S .
$$

This gives

$$
g^{\epsilon}(\boldsymbol{\theta}) \rightarrow g^{0}(\boldsymbol{\theta})=-\frac{1}{2} \int_{\Omega_{\epsilon}^{ \pm}} \operatorname{Tr}\left(\boldsymbol{\sigma}^{\mathbf{0}} \cdot \boldsymbol{\nabla} \boldsymbol{u}^{\mathbf{0}}\right) \operatorname{div} \boldsymbol{\theta} d \Omega+\int_{\Omega_{\epsilon}^{ \pm}} \operatorname{Tr}\left(\boldsymbol{\sigma}^{\mathbf{0}} \cdot \boldsymbol{\nabla} \boldsymbol{u}^{\mathbf{0}} \cdot \boldsymbol{\nabla} \boldsymbol{\theta}\right) d \Omega
$$

On the other hand, $\epsilon$ being fi xed, it was mentioned previously that the local energy release rate given by the expression (22) is always strictly positive, even if there is not a crack. It is tempting to take this value as the value for crack initiation. Theorem 1 indicates that it depends on the joint thickness and tends to zero with $\epsilon$. As a consequence, it cannot be taken as crack initiation value. It is now admitted that crack initiation can be detected using a maximum tensile stress criterion of the form $\sigma_{33}=\sigma_{33 c}$. However, for a given thickness of the joint, the stress criterion, the maximum jump of the normal displacement criterion $\left(\left[u_{3}\right]=\left[u_{3 c}\right]\right)$ and the debonding force criterion $\left(G=G_{c}\right)$ are equivalent. For example, in the case of a DCB specimen in small displacement, the constitutive equations (7) and the expression (22) give :

$$
\left[u_{3 c}\right]=\frac{\epsilon h}{R_{3333}^{m}} \sigma_{33 c} \quad ; \quad G_{I c}=\frac{\epsilon h}{2 R_{3333}^{m}}\left(\sigma_{33 c}\right)^{2} .
$$

The link between these criterions is studied experimentally in (Chai (1986)) and theoretically in (Leguillon (2002)). 


\subsection{Connection with the damage interface models}

It is now common to use damage interface models to simulate delamination growth in layered composite structures (Ladevèze (1992), Allix and Corigliano (1999), Alfano and Crisfi eld (2001)). Among these models, one of the most used is the Tvergaard's model (Tvergaard (1990), Chaboche et al. (2001)) which considers a zero thickness interface equipped, for example in the case of a DCB specimen submitted to a monotone loading in mode I, with the following constitutive equation :

$$
T_{3}^{t v e r}=\frac{27}{4} \sigma_{\max } \frac{\left[u_{3}\right]}{\left[u_{3 c}^{\text {tver }}\right]}(1-d)^{2} \quad ; \quad d=\frac{\left[u_{3}\right]}{\left[u_{3 c}^{\text {tver }}\right]} .
$$

Here, the models parameters $\sigma_{\max }$ and $\left[u_{3 c}^{\text {tver }}\right]$ are the maximum tensile stress that the interface can support and the normal displacement jump at fracture respectively, whereas $d \in[0,1]$ is a decohesion indicator viewed as a damage parameter equal to zero for a perfectly bonded interface and equal to one for a broken interface. The two parameters are connected writing that the area under the loading curve is equal to the critical energy release rate $G_{I C}$ :

$$
\int_{0}^{\left[u_{3 c}^{\text {tver }}\right]} \frac{27}{4} \sigma_{\max } \frac{\left[u_{3}\right]}{\left[u_{3 c}^{\text {tver }}\right]}\left(1-\frac{\left[u_{3}\right]}{\left[u_{3 c}^{\text {tver }}\right]}\right)^{2} d\left[u_{3}\right]=G_{I c} .
$$

This gives :

$$
\sigma_{\max }=\frac{16}{9} \frac{G_{I c}}{\left[u_{3 c}^{t v e r}\right]}
$$

In fact, the interface between two lamina is not a perfect interface but rather a thin matrix layer of thickness $e$. In the present case, the debonding force derived from the expression (12) takes the following form :

$$
G=\frac{1}{2} \frac{R_{3333}^{m}}{e}\left(\left[u_{3}\right]+\frac{1}{2 e}\left[u_{3}\right]^{2}\right)^{2} .
$$

Then, the Griffi th's criterion $G=G_{I c}$ gives the following expression of the normal displacement jump at debonding :

$$
\left[u_{3 c}\right]=-e+e \sqrt{1+\sqrt{\frac{8 G_{I c}}{R_{3333}^{m} e}}}=O\left(e^{\frac{1}{2}}\right) .
$$

Then, assuming that debonding occurs for the same value of the normal displacement jump $\left(\left[u_{3 c}^{\text {tver }}\right]=\right.$ $\left.\left[u_{3} c\right]\right)$, the parameters of the Tvergaard's model can be identifi ed. The loading curves of both the models are reported in the fi gure 4: the coeffi cient have been identifi ed in order that the two models are energetically equivalent, and the complete decohesion occurs for the same jump $\delta_{3}$. 


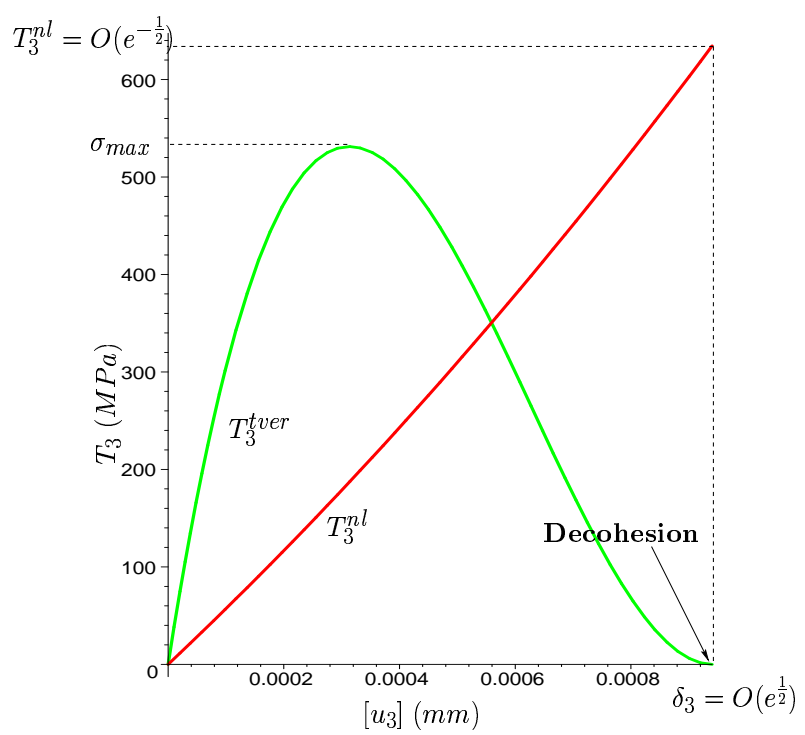

Figure 4: $T_{3}^{t v e r}$ and $T_{3}^{n l}$ vs. $\left[u_{3}\right]$.

With the numerical values $\epsilon h=0.1 \mathrm{~mm}, R_{3333}^{m}=4000 \mathrm{MPa}$ and $G_{c}=0.281 \mathrm{~N} / \mathrm{mm}$, we obtain $\left[u_{3 c}\right]=\left[u_{3 c}^{t v e r}\right] \approx 0.00094 \mathrm{~mm}$ and $\sigma_{\max } \approx 531.19 \mathrm{MPa}$. For the springs layer model, the critical traction of decohesion is $T_{3} \approx 560.71 \mathrm{MPa}$. However, when irreversible mechanisms can be neglected, cohesive models should be avoided for at least two reasons : fi rstly, their numerical resolution leads to serious mesh dependencies both noted in (Alfano and Crisfi eld (2001)) and (Chaboche et al. (2001)). In order to solve this problem, very fi ne meshes are needed near the crack front. On contrary, the use of the $\theta$-method to obtain the energy release rate, a global quantity, with the relation (21), allow to use relatively coarse meshes. Secondly, the strong nonlinear character of the cohesive law requires a larger number of iteration of a Newton's method. Numerical comparisons has been performed on structural examples (Roudolff and Ousset (2002)) and confi rmed this last point.

\section{Crack growth algorithm}

\subsection{Numerical approximation of the displacement field}

In this paragraph, we describe the discretization used for the displacement fi eld and give the tangent stiffness matrix in the joint associated to the Newton's method on the equation (12). A sixteen nodes fi nite element is used: the shape function are quadratic with respect to the in-plane variables and linear with respect to the out-of-plane variable. In the adherents $\Omega_{\epsilon}^{ \pm}$, and in the joint $\Omega_{\epsilon}^{m}$ associated to the 
complete model, the $i$-th component displacement fi eld is written as follow :

$$
u_{i}(\eta, \xi, \zeta)=\sum_{k=1}^{8} p_{k}(\eta, \xi)\left[\frac{1+\zeta}{2} u_{i}^{k S}+\frac{1-\zeta}{2} u_{i}^{k I}\right], \quad i=1,3 ; \quad(\eta, \xi, \zeta) \in[-1,1]^{3}
$$

$p_{k}$ are the in-plane shape function and $u_{i}^{k S}$ (resp. $u_{i}^{k I}$ ) the $i$-th displacement component of the node $n^{k S}$ (resp. $\left.n^{k I}\right), k=1,8$ located at the upper interface $\zeta_{1}=1$ (resp. lower interface $\zeta=-1$ ). When the simplifi ed model is used, the joint is replaced by its mid-surface and the nodes $n^{k S}$ and $n^{k I}$, are, in the reference confi guration, geometrically merged in the plane $\zeta=0$. The displacement jump is then discretized in the following way:

$$
\left[u_{i}\right](\eta, \xi, 0) \equiv u_{i}^{S}(\eta, \xi, 0)-u_{i}^{I}(\eta, \xi, 0)=\sum_{k=1}^{8} p_{k}(\eta, \xi)\left(u_{i}^{k S}-u_{i}^{k I}\right), \quad i=1,3
$$

and imply that, for $i, j=1,3$ :

$$
\left(\left[u_{i}\right]\left[v_{j}\right]\right)(\eta, \xi, 0)=\sum_{m, n=1}^{8} p_{m}(\eta, \xi) p_{n}(\eta, \xi)\left(\begin{array}{c}
v_{j}^{n I} \\
v_{j}^{n S}
\end{array}\right)^{t}\left(\begin{array}{cc}
1 & -1 \\
-1 & 1
\end{array}\right)\left(\begin{array}{c}
u_{i}^{m I} \\
u_{i}^{m} S
\end{array}\right) .
$$

Then, the Newton's equation associated to the equation (12) is, for all $v \in \tilde{V}\left(\Omega^{ \pm}\right)$:

$$
\begin{aligned}
T\left(\boldsymbol{u}^{\boldsymbol{n}}, \boldsymbol{v}\right) \cdot \boldsymbol{w}= & \lambda \int_{\Gamma_{f}^{ \pm}} \boldsymbol{f}^{\epsilon} \cdot \boldsymbol{v} d \Gamma-\int_{\Omega_{\epsilon}^{ \pm}} \operatorname{Tr}\left(\mathbb{R}^{ \pm}: \boldsymbol{\nabla} \boldsymbol{u}^{\boldsymbol{n}} \cdot \boldsymbol{\nabla} \boldsymbol{v}\right) d \Omega \\
& -\int_{S}\left[\sigma_{\alpha 3}\left(\boldsymbol{u}^{\boldsymbol{n}}\right)\left[v_{\alpha}\right]+\sigma_{33}\left(u^{n}\right)\left(\left[v_{3}\right]+\frac{1}{\epsilon h}\left[u_{k}^{n}\right]\left[v_{k}\right]\right)\right] d S
\end{aligned}
$$

where $\boldsymbol{w} \equiv \boldsymbol{u}^{\boldsymbol{n + 1}}-\boldsymbol{u}^{\boldsymbol{n}}$ and $n$ is the index of the incremental method. If $\{\boldsymbol{v}\}$ is the vector of the nodal value of length $48\{\boldsymbol{v}\}=\left\{v^{1}, v^{2}, \ldots, v^{8}\right\}$ with $v^{j}=\left(v_{1}^{j I}, v_{2}^{j I}, v_{3}^{j I}, v_{1}^{j S}, v_{2}^{j S}, v_{3}^{j S}\right)$, then the approximations of the integrals in the left hand side of (43) take the form :

$$
\{\boldsymbol{v}\}^{t} \cdot\left(\int_{S}[B] d S\right) \cdot\{\boldsymbol{w}\}
$$

where the symmetric matrix $[B]$ is made of symmetric matrices $\left[C_{i j}\right]_{6 \times 6}$ of order 6 such that :

$$
B(6(i-1)+1: 6 i, 6(j-1)+1: 6 j)=C_{i j}(1: 6,1: 6), \quad i, j=1,8
$$

with

$$
\left[C_{i j}\right]=p_{i}(\eta, \xi) p_{j}(\eta, \xi)\left(\begin{array}{cc}
{[A]_{3 \times 3}} & -[A]_{3 \times 3} \\
-[A]_{3 \times 3} & {[A]_{3 \times 3}}
\end{array}\right)
$$


and

$$
\left\{\begin{array}{c}
A_{l l}=\frac{R_{l 3 l 3}^{m}}{\epsilon h}+\frac{R_{l 3 l 3}^{m}}{\epsilon h} \frac{\left[u_{l}\right]^{2}}{(\epsilon h)^{2}}+\frac{R_{3333}^{m}}{\epsilon h}\left(\left(1+2 \delta_{l 3}\right) \frac{\left[u_{3}\right]}{\epsilon h}+\frac{1}{2} \frac{\left[u_{k}\right]\left[u_{k}\right]}{(\epsilon h)^{2}}\right), \quad l=1,3 \\
A_{l m}=A_{m l}=\frac{R_{3333}^{m}}{\epsilon h} \frac{\left[u_{l}\right]}{\epsilon h}\left(\delta_{m 3}+\frac{\left[u_{m}\right]}{\epsilon h}\right) \quad l, m=1,3 \quad l \neq m .
\end{array}\right.
$$

( $\delta_{i j}$ is the Kronecker's symbol).

\subsection{Approximation of the crack front and definition of the $\theta$ field}

The front $c_{f}$ is approximated using $B_{3}$-spline functions. This allows us to disconnect the $c_{f}$ description from the fi nite element mesh. The curve $c_{f}$ is described by nodes $x^{i}$ and the part of $c_{f}$ located between two nodes constitutes a spline element. Then, the parametric equation of the $k$-th element is :

$$
x(\tau)=\sum_{i=1}^{4} p_{i}(\tau) \phi_{x}^{i+k-1},
$$

where $\phi_{x}^{i}$ are the geometrical spline coordinates and $\tau$ is the curvilinear abscissa along $c_{f}$. Finally, in the neighbourhood of the $k$-th spline element, $\boldsymbol{\theta}$ is defi ned as :

$$
\boldsymbol{\theta}(\tau, \rho)=q(\rho) \sum_{i=1}^{4} p_{i}(\tau) \boldsymbol{\phi}_{\boldsymbol{\theta}}^{\boldsymbol{i}+\boldsymbol{k}-\mathbf{1}}
$$

where $\rho$ is the transverse local coordinate and $q$ is a smooth bell-shaped function equal to 1 for $\rho=0$ and 0 for $\rho=\rho_{r}$. Let $\boldsymbol{M}$ be a point of $S, \boldsymbol{m}$ be its orthogonal projection onto $c_{f}$ and $\boldsymbol{\nu}$ the unit normal to $c_{f}$ at $\boldsymbol{m}$, then $\rho$ is defi ned by the relation :

$$
O M=O m+\rho \boldsymbol{\nu} .
$$

The $\boldsymbol{\theta}$ degrees of freedom are the spline coordinates $\phi_{\theta}$. Generally, their number is lower than the number of all the nodes of the fi nite element mesh located on $c_{f}$, reducing in this way the size of the problems (20) and (29).

The discretized form of relation (21) and (25 ) can be written as $\{\boldsymbol{\theta}\}^{t} \cdot\{\boldsymbol{g}\}$ and $\{\boldsymbol{\theta}\}^{t} \cdot\left\{\boldsymbol{g}_{\boldsymbol{c}}\right\}$ respectively whereas the right-hand side of problem (20) is discretized as :

$$
\{\boldsymbol{v}\}^{t} \cdot[\boldsymbol{F}] \cdot\{\boldsymbol{\theta}\}>
$$

Finally, the second derivatives $(24)$ and $\left(25_{2}\right)$ take the following forms :

$$
\{\boldsymbol{\theta}\}^{t} \cdot\left[J^{(2)}\right] \cdot\{\boldsymbol{\theta}\} \quad ; \quad\{\boldsymbol{\theta}\}^{t} \cdot\left[D^{(2)}\right] .\{\boldsymbol{\theta}\},
$$

where $\left[J^{(2)}\right]$ and $\left[D^{(2)}\right]$ are symmetric matrices, $\left[J^{(2)}\right]$ being fully populated. 


\subsection{Debonding algorithm}

The debonding algorithm is described in the case of displacement control. Let

- $\left[K_{T}\right]$ be the tangent stiffness matrix ;

- $\{R\}$ be the vector of internal residual forces ;

- $\lambda$ be the loading factor ;

- $j$ the number of the control degree of freedom;

- $[Q]$ be the matrix of the eigenvectors of $\left[D^{(2)}\right]$ associated to positive eigenvalues ;

then, the algorithm is as follow : 
loop $n$ : control displacement increment $u_{d}^{n}$,

loop $k:$ front increment $\gamma_{f}^{n, k}$,

loop $i$ : equilibrium equations,

$$
\begin{aligned}
& \left\{\Delta u^{i}\right\}=\left\{\Delta u_{R}^{n, k, i}\right\}+\Delta \lambda^{i}\left\{u_{f}^{n, k}\right\}, \\
& {\left[K_{T}^{n, k}\right] \cdot\left\{\Delta u_{R}^{n, k, i}\right\}=-\left\{R^{n, k, i}\right\}+\lambda^{n, k, i}\{f\},} \\
& {\left[K_{T}^{n, k}\right] .\left\{u_{f}^{n, k}\right\}=\{f\},} \\
& \qquad \Delta \lambda^{i}=-\frac{\left\{\Delta u_{R}^{n, k, i}\right\}_{j}}{\left\{u_{f}^{n, k}\right\}_{j}}, \\
& \left\{u^{n, k, i+1}\right\}=\left\{u^{n, k, i}\right\}+\left\{\Delta u^{i}\right\}, \\
& \lambda^{n, k, i+1}=\lambda^{n, k, i}+\Delta \lambda^{i},
\end{aligned}
$$

if $\left\|\left\{R^{n, k, i+1}\right\}\right\|_{L^{\infty}(\mathbb{R})} \leq \epsilon_{R}$, end of loop $i$,

compute $\left[K_{T}^{n, k}\right]$ at the new equilibrium point,

compute $\{\boldsymbol{g}\}$ and $\left\{\boldsymbol{g}_{\boldsymbol{c}}\right\}$,

compute $\left[\boldsymbol{u}^{(\mathbf{1})}\right]$ solving $\left[K_{T}^{n, k}\right] .\left[\boldsymbol{u}^{(\mathbf{1})}\right]=\left[F^{n, k}\right]$,

compute $\left[J^{(2)}\right],\left[D^{(2)}\right],[Q]$,

compute $\{\boldsymbol{\Theta}\}$ solving :

$$
\left([Q]^{t} \cdot\left[E^{(2)}\right] \cdot[Q]\right) \cdot\{\boldsymbol{\Phi}\}=[Q]^{t} \cdot\left\{\boldsymbol{g}-\boldsymbol{g}_{\boldsymbol{c}}\right\} \quad ; \quad\{\boldsymbol{\Theta}\}=[Q] .\{\boldsymbol{\Phi}\},
$$

if $\|\{\Theta\}\|_{L^{\infty}(\mathbb{R})}>\epsilon_{\theta}$ move the front:

$$
\gamma_{f}^{n, k+1}: x^{n, k+1}=x^{n, k}+\epsilon_{S} \Theta\left(x^{n, k}\right),
$$

remeshing and go to loop $i$,

if $\|\{\boldsymbol{\Theta}\}\|_{L^{\infty}(\mathbb{R})} \leq \epsilon_{\theta}$ end of loop $k$,

$u_{d}^{n+1}=u_{d}^{n}+\Delta u_{d}^{n}$,

end of loop $n$.

This algorithm can be repeated until $c_{f} \subset \partial S$, i.e. when the mid-surface of the joint is completely broken and the two adherents are disconnected. Before we give some applications, let us make two comments with regard to the present algorithm:

1. There are several ways to compute the new displacement fi eld after remeshing. Here, we have presented the case where the new equilibrium point is sought starting from the displacements obtained with the previous mesh. Unfortunately, if the front displacement is too large, the equilibrium cannot be recovered. To prevent divergence, small increments of the front displacement must be made: this is the role of the real $\left.\left.\epsilon_{S} \in\right] 0,1\right]$. Another way is to restart the computation from unloading. This way seems more costly, but it allows greater displacement front increments. 
2. The remeshing step can be time consuming for complex structures. Some authors have recently proposed a method without remeshing where the description of the crack front is independent of the mesh structure (Moes et al. (1999), Wells and Sluys (2001)).

\section{Numerical applications}

\subsection{Analytical solution for the linear beam problem}

The fi rst application concerns the analytical resolution of the beam problem: two homogeneous and isotropic beams of thickness $\eta$ are bonded together by an adhesive of thickness $\epsilon h$. The assembly is clamped at $x=0$ and is submitted to a normal displacement $\delta$ at the end of the lower and upper delaminated arms at $x=L$ (see Figure 5).

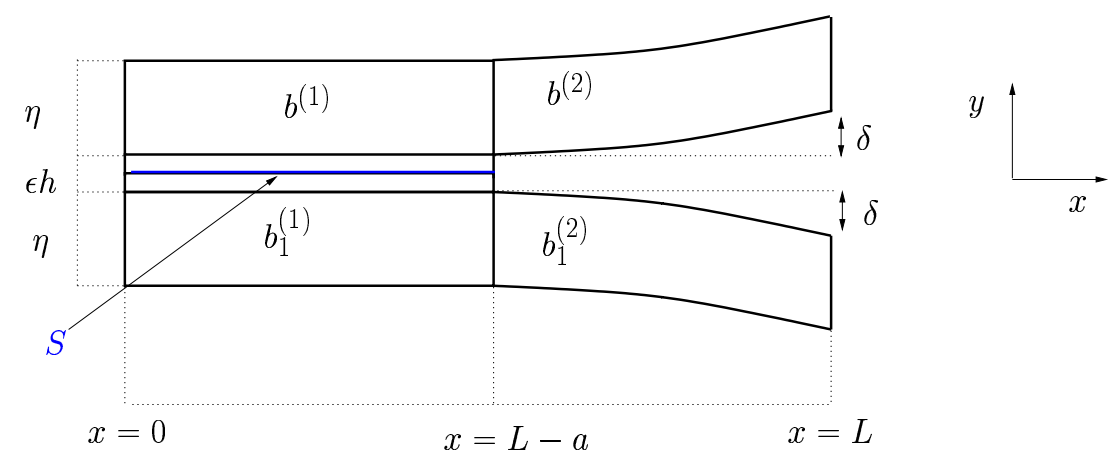

Figure 5: The beam problem.

As in the previous sections, the adhesive is modelled by its mid-line $S$. Thanks to the symmetry of the problem, the jump $\left[u_{1}\right]$ across $S$ is zero and the energy of the problem is (to simplify, we neglect the large displacement) :

$$
J=\int_{0}^{L-a} m^{(1)} \frac{d^{2} u_{2}^{(1)}}{d x^{2}} d x+\int_{L-a}^{L} m^{(2)} \frac{d^{2} u_{2}^{(2)}}{d x^{2}} d x+\frac{1}{2} \frac{E_{3}}{\epsilon h} \int_{0}^{L-a}\left[u_{2}\right]^{2} d x
$$

with the flexural moment $m^{(i)}=\frac{E_{i} \eta^{3}}{12} \frac{d^{2} u_{2}}{d x^{2}}$ and $E_{i}$ the Young's modulus of the $i$-th beam and $E_{3}$ the Young's modulus of the joint. The displacements verify the following differential equations :

$$
\left.\frac{d^{4} u_{2}^{(1)}}{d x^{4}}+\frac{12 E_{3}}{\epsilon h E_{1} \eta^{3}} u_{2}^{(1)}=0 \quad x \in\right] 0, L-a\left[\quad ; \quad \frac{d^{4} u_{2}^{(2)}}{d x^{4}}=0 \quad x \in\right] L-a, L[
$$


showing that the displacement $u_{2}^{(1)}\left(\right.$ resp. $\left.u_{2}^{(2)}\right)$ is generated by the basis $\left\{e^{ \pm \alpha x} \cos (\alpha x), e^{ \pm \alpha x} \sin (\alpha x)\right\}$, $\alpha=\left(\frac{6 E_{3}}{\epsilon h E_{1} \eta^{3}}\right)^{\frac{1}{4}}$ (resp. $\left\{1, x, x^{2}, x^{3}\right\}$ ). The fi gure 6 depicts the evolution of the displacement $u_{2}^{(1)} \equiv$ $\frac{1}{2}\left[u_{2}^{(1)}\right]$ for several values of $\epsilon$ and $a=40 \mathrm{~mm}, L=80 \mathrm{~mm}, \delta=1 \mathrm{~mm}, E_{1}=E_{2}=150000 \mathrm{MPa}, E_{3}=$ $4000 \mathrm{MPa}, \eta=4 \mathrm{~mm}$ and $h=10 \mathrm{~mm}$.
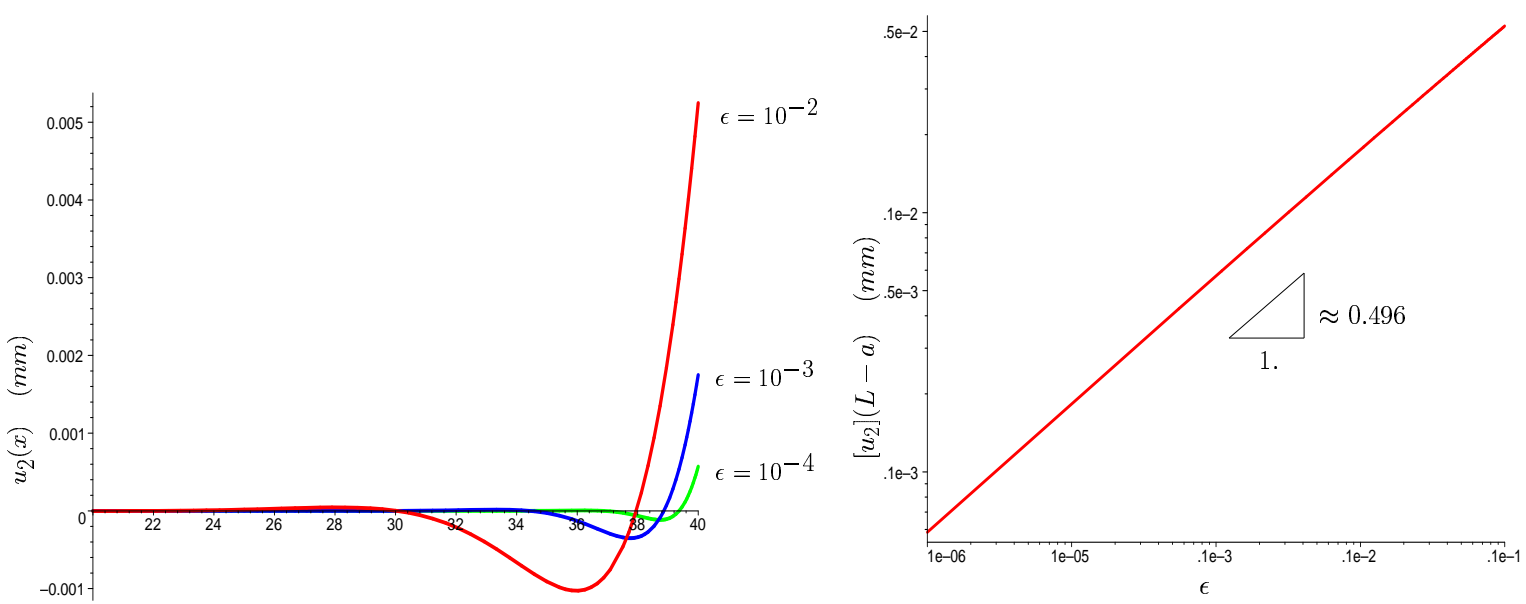

Figure 6: $u_{2}(x) \equiv \frac{1}{2}\left[u_{2}\right](x), x \in[20, L-a] . \quad$ Figure 7: $\left[u_{2}\right](L-a)$ vs. $\epsilon$ in a loglog scale.

The jump $\left[u_{2}\right]$ presents some oscillations near the crack front induced by the elastic joint. At some points of $S$, the jump is negative ; however, the orientation-preserving condition (9) is satisfi ed. The energy release rate, calculated for $a$ large enough, is a decreasing function of $a$ : the propagation of the crack is stable. When $\epsilon$ tends to zero, the model reproduces the results of fracture mechanics theory for a perfectly bonded interface. On this example, for $x \leq L-a$, the jump $\left[u_{2}\right]$ converges to zero as approximatively $\sqrt{\epsilon}:\left[u_{2}\right](x)=O\left(\epsilon^{0.496}\right)$ (see fi gure 7 for $x=L-a$ ) and the energy release rate $g$ to the fracture mechanics ones $g_{F M}$ as approximatively $\epsilon^{\frac{1}{4}}: g_{F M}(x)-g(x)=O\left(\epsilon^{\frac{1}{4}}\right)$.

\subsection{Opening debonding}

The second example was devoted to the study of a double cantilever beam of length $L$ and width $l$ (see fi gure 1) where the crack is initially straight $c_{f}=\left\{\boldsymbol{x} \in S, x_{1}=L-a\right\}, a \in[0, L]$. The structure was clamped on the side $\Gamma_{u}=\left\{\boldsymbol{x} \in \Omega_{\epsilon}, x_{1}=0\right\}$ and submitted to normal load $\boldsymbol{f}=\left(0,0, f_{3}^{ \pm}\right), f_{3}^{+}=-f_{3}^{-}$, on the opposite sides $\left\{x_{1}=L\right\}$. The modifi ed Newton's algorithm was displacement controlled and let $u_{d}$ be the normal displacement at the end of the upper delaminated arm. The constituents are made of isotropic homogeneous materials and the characteristics of the assembly are reported in the table 1. The fi gure 8 compares, for $a=40 \mathrm{~mm}$, the energy release rate along the front $c_{f}$, obtained from the relation (21) (curve with $\circ$ symbol) and the relation (22) (curve with + symbol) to the energy 


\begin{tabular}{ccccccccc}
\hline$E^{ \pm}$ & $\nu^{ \pm}$ & $E^{m}$ & $\nu^{m}$ & $L$ & $l$ & $h$ & $\epsilon h$ & $G_{c}$ \\
\hline $150000 M P a$ & 0.3 & $4000 M P a$ & 0.35 & $80 \mathrm{~mm}$ & $40 \mathrm{~mm}$ & $4 m \mathrm{~m}$ & $0.1 \mathrm{~mm}$ & $0.373 \mathrm{~N} / \mathrm{mm}$ \\
\hline
\end{tabular}

Table 1: Material and geometrical characteristics.

release rate along the front $C_{f} \cap S^{+}$obtained from the complete model (curve with $*$ symbol). The three computations are very close. Varying the parameter $\epsilon$, we obtain that the energy release rate $g^{\epsilon}$ obtained from the complete model converges toward $g$ obtained from (21) as $\epsilon$ showing, on this example, that the simplifi ed model is of order one: this result is in good agreement with the used asymptotic expansion (5). Let us remark that the energy release rate is approximately constant along the front except near the edges.
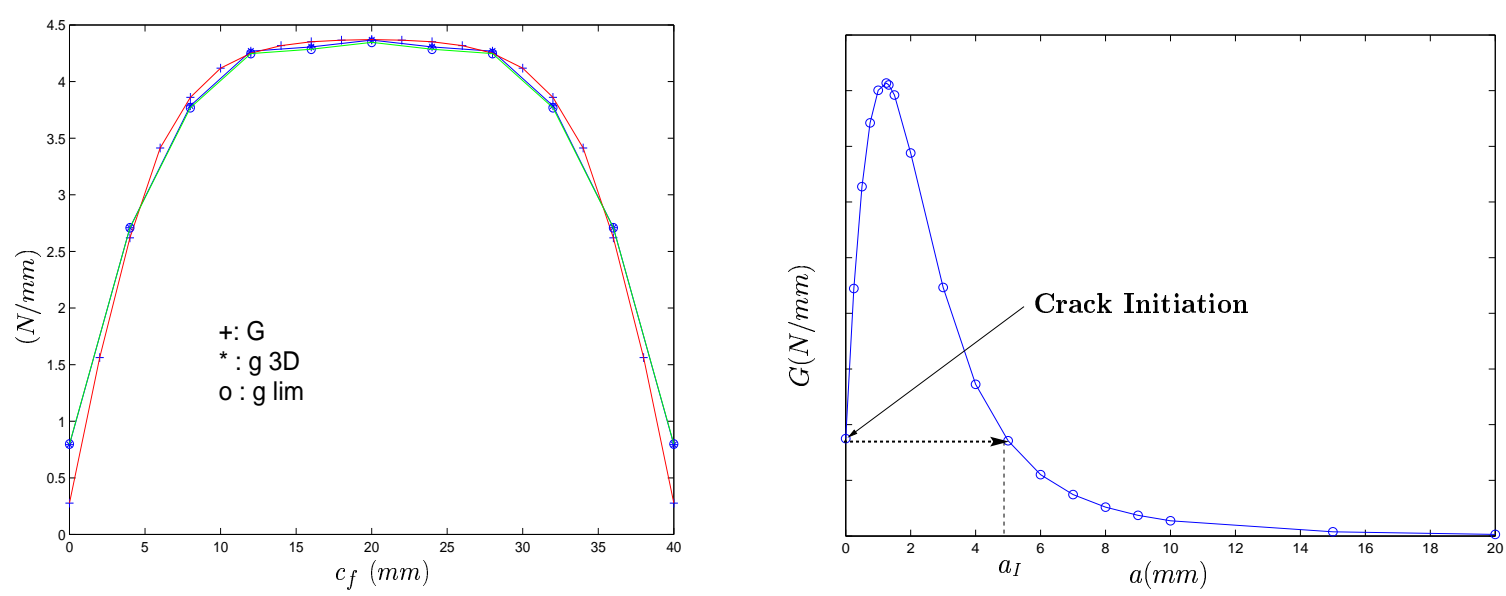

Figure 8: Local energy release rate along $c_{f}$. Figure 9: Delamination force $G\left(L-a, \frac{l}{2}\right)$ vs. a.

The fi gure 9 shows the typical variations of the delamination force $G$ (expression 23) at the point $\left(x_{1}, x_{2}\right)=\left(L-a, \frac{l}{2}\right)$ of the front with respect to the delamination length $a$. For $a=0, G$ is strictly positive; it increases fi rst with $a$ and then it decreases. As a consequence, the crack growth just after initiation is unstable. The growth stability is recovered once the crack length has reached the value $a_{I}$. Here, $a_{I}$ ranges in the interval $[4 . \mathrm{mm}, 6 . \mathrm{mm}]$, whereas the value $u_{d c}$ of the control variable $u_{d}$ at crack initiation is taken equal arbitrarily to $0.1 \mathrm{~mm}$. When $a$ is large enough (approximatively three or four times the thickness of the joint), the delamination force becomes lower than its initial value. Let us now take arbitrarily the delamination $G_{c}$ equal to $0.373 \mathrm{~N} / \mathrm{mm}$. The delamination force, obtained from the relation (22) reaches this value along the front, except on the border where $g$ is slightly smaller, when the imposed displacement $u_{d}$ reach the critical value $u_{d c}=0.01 \mathrm{~mm}$. The fi gure similar to fi gure 9 indicates that the length $a_{I}$ of the front $c_{f}$, approximately constant along $c_{f}$ is in the ranges $[2.5 \mathrm{~mm}, 3 \mathrm{~mm}]$. Then, the algorithm of propagation is used, starting with $u_{d}=u_{d c}+\eta$ and a straight 
crack of length lower than $2.5 \mathrm{~mm}$, for instance $1 \mathrm{~mm}$. The different front locations are reported in the fi gure 10 for $u_{d}$ ranging from $0.01 \mathrm{~mm}$ to $0.06 \mathrm{~mm}$.

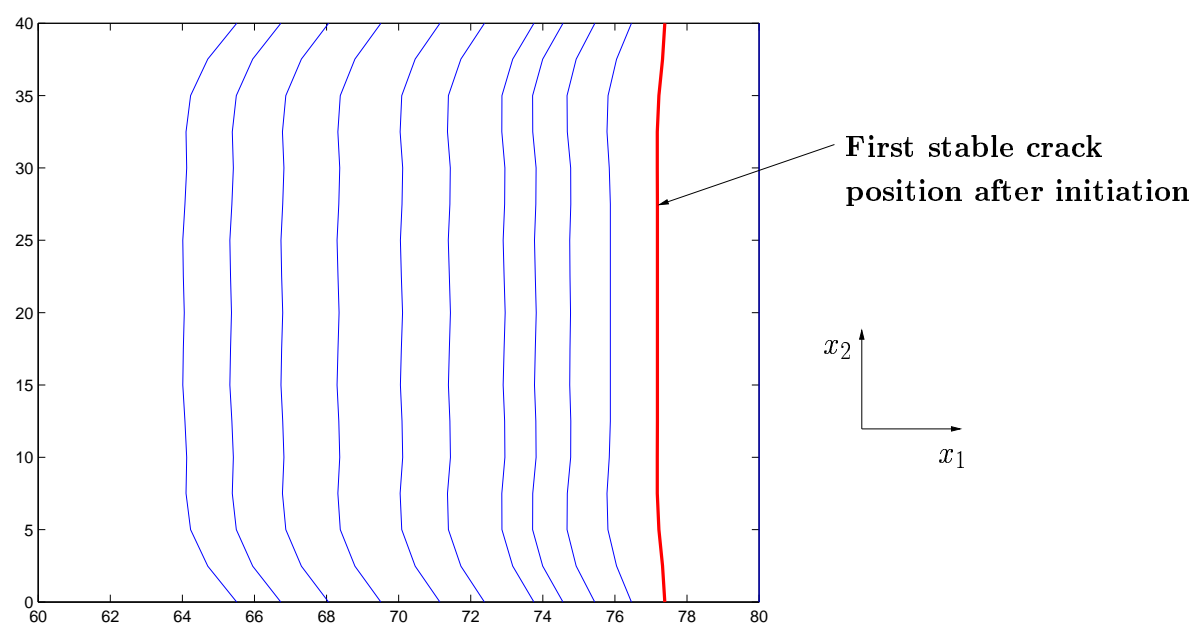

Figure 10: Front positions for $u_{d}$ from $0.01 \mathrm{~mm}$ to $0.06 \mathrm{~mm}$.

As expected, the front was curving near the edges of the specimen during the growth. The critical values at initiation, including the strain $\gamma_{33 c}=\frac{\left[u_{3}\right]_{c}}{\epsilon h}+\frac{1}{2} \frac{\left[u_{k}\right]_{c}\left[u_{k}\right]_{c}}{(\epsilon h)^{2}}$, are reported in the table 2. Figures 11 and 12 represent the face $x_{1}=L$ of the specimen just before and after the crack initiation.
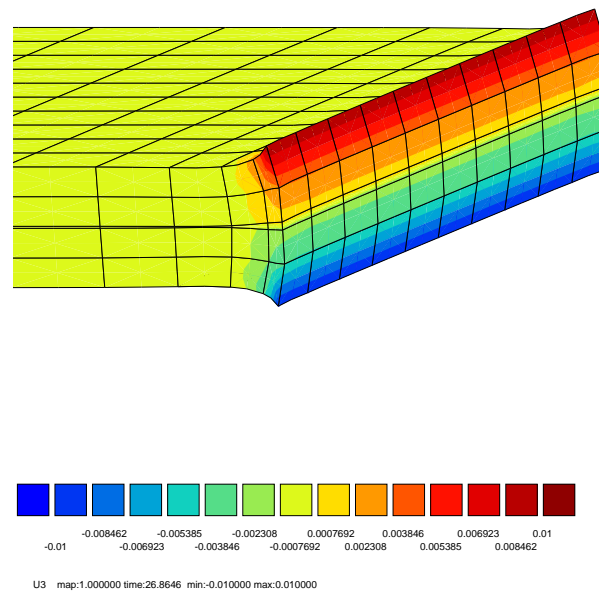

Figure 11: $u_{3}$ with $u_{d}=0.01 \mathrm{~mm}$.
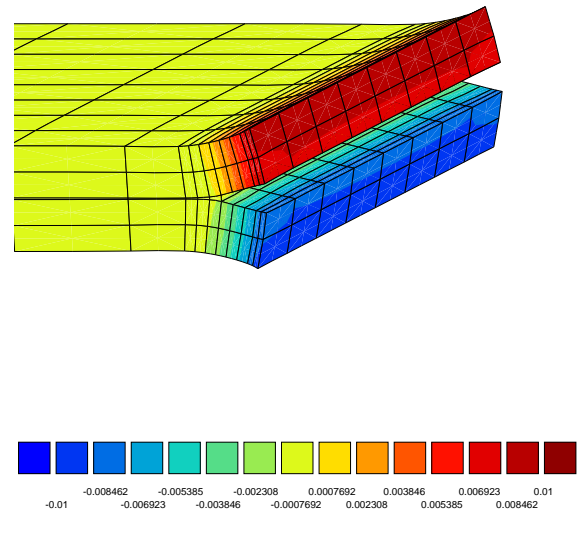

Figure 12: $u_{3}$ with $u_{d}=0.01 m m+\eta$. 


\begin{tabular}{ccccc}
\hline$u_{d c}$ & {$\left[u_{3}\right]_{c}$} & $G_{c}$ & $\sigma_{33 c}$ & $\gamma_{33 c}$ \\
\hline $0.01 \mathrm{~mm}$ & $0.0033 \mathrm{~mm}$ & $0.373 \mathrm{~N} / \mathrm{mm}$ & $218.89 \mathrm{MPa}$ & $3.5 \%$ \\
\hline
\end{tabular}

Table 2: Critical values on initiation.

The variations of the loading factor $\lambda$ with respect to the control variable $u_{d}$ are reported in the fi gure 13 for two values of the control variable increment $\delta u_{d}$. The point with the same abscissa correspond to iterations on the front location (loop $k$ in the algorithm). The convergence in the loop $k$ is obtained in four iterations for $\delta=0.005 \mathrm{~mm}$ and in twelve iterations for $\delta u_{d}=0.05 \mathrm{~mm}$. Whatever the value of the increment $\delta u_{d}$, the equilibrium points of $E$ in respect to the front location and to the displacement fi eld are on the same curve.

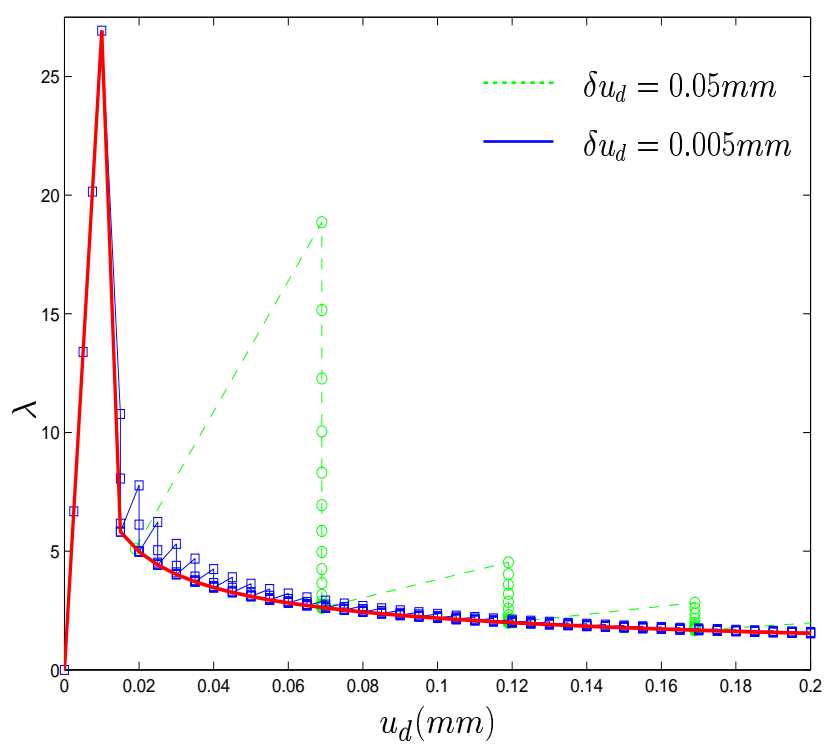

Figure 13: Loading factor $\lambda$ vs. $u_{d}$.

In this numerical example, an arbitrarily value of the critical energy release rate $G_{c}$ has been taken in the interval $[0.3 \mathrm{~N} / \mathrm{mm}, 0.4 \mathrm{~N} / \mathrm{mm}]$. This interval of value is usually obtained for composite structures without joint (see [Robinson and Song (1992)] for the DCB). For this interval, large values of the strain $\gamma_{33 c}$, predominant here, are obtained (Table 2). This shows the presence of large displacement. For bonded assemblies, this situation is accentuated : indeed, the critical energy release rate is an increasing function of the softness of the adhesive (Chai (1986)) : this point signifi es that for usual soft adhesive, an important loading factor is required in order that the delamination force $G$ (23), 
which is, for opening mode, an increasing function of the jump $\left[u_{l}\right]$ across $S$, reach the value $G_{c}$. If we note $G^{l}=\frac{1}{2 \epsilon h} R_{l 3 l 3}^{m}\left[u_{l}\right]^{2}$ the delamination force obtained with the Hooke's law and if we neglect $\left[u_{1}\right]$ and $\left[u_{2}\right]$ with respect to $\left[u_{3}\right]$, we have the relative difference

$$
\frac{G-G^{l}}{G^{l}}=\frac{\left[u_{3}\right]}{\epsilon h}+\frac{1}{4}\left(\frac{\left[u_{3}\right]}{\epsilon h}\right)^{2} \approx \gamma_{33},
$$

showing that the difference on the energy release rate between Hooke's law and nonlinear law derived from the stored energy function of St-Venant Kirchhoff is very important for soft adhesive. Let us add, due to the inequality $G>G^{l}$, that the critical loading factor, observed just before fracture, is overestimated with the Hooke's law. As a consequence, the nonlinear law permits to anticipate better the fracture of the joint.

The following results illustrate the comments made in the paragraph 4.1. To this end, the computations were made for two different values of the joint thickness. The fi gures 14 and 15 depict respectively the jump $\left[u_{3}\right]$ and $\sigma_{33}$ respectively at the point $\left(L-a, \frac{l}{2}\right)$ of the front $c_{f}$ as a function of the crack length $a$, whereas the values of the energy release rate are reported in the fi gure (16).

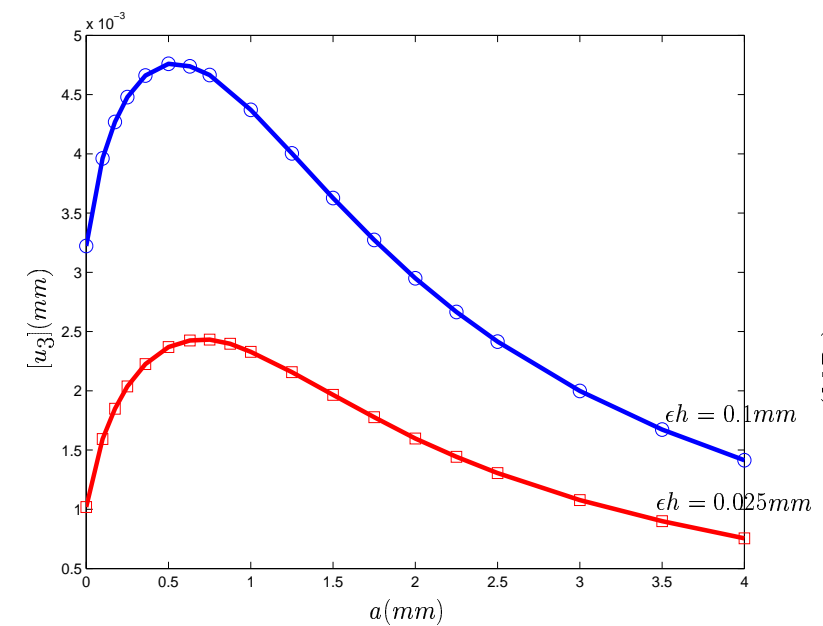

Figure 14: $\left[u_{3}\right]\left(L-a, \frac{l}{2}\right)$ vs. $a$.

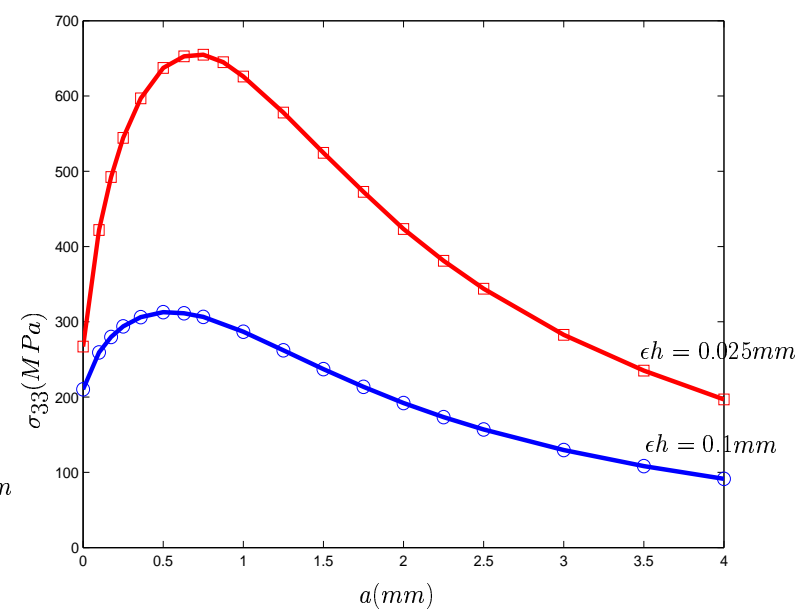

Figure 15: $\sigma_{33}\left(L-a, \frac{l}{2}\right)$ vs. $a$ in the joint.

When the thickness of the joint tends to zero, the jump converge as expected to zero, and the stress becomes singular, except on $a=0$ (no crack) where the stress, noted $\sigma_{33}(0)$ is fi nite. The curve " $\epsilon h=0$ " on fi gure 16 corresponds to the energy release rate of the perfectly bonded model (expression (33)) and is prolonged by continuity at the origin by zero. In view of this fi gure, it seems that the slopes of the different curves near the origin have similar values. This point would be worth studying. 


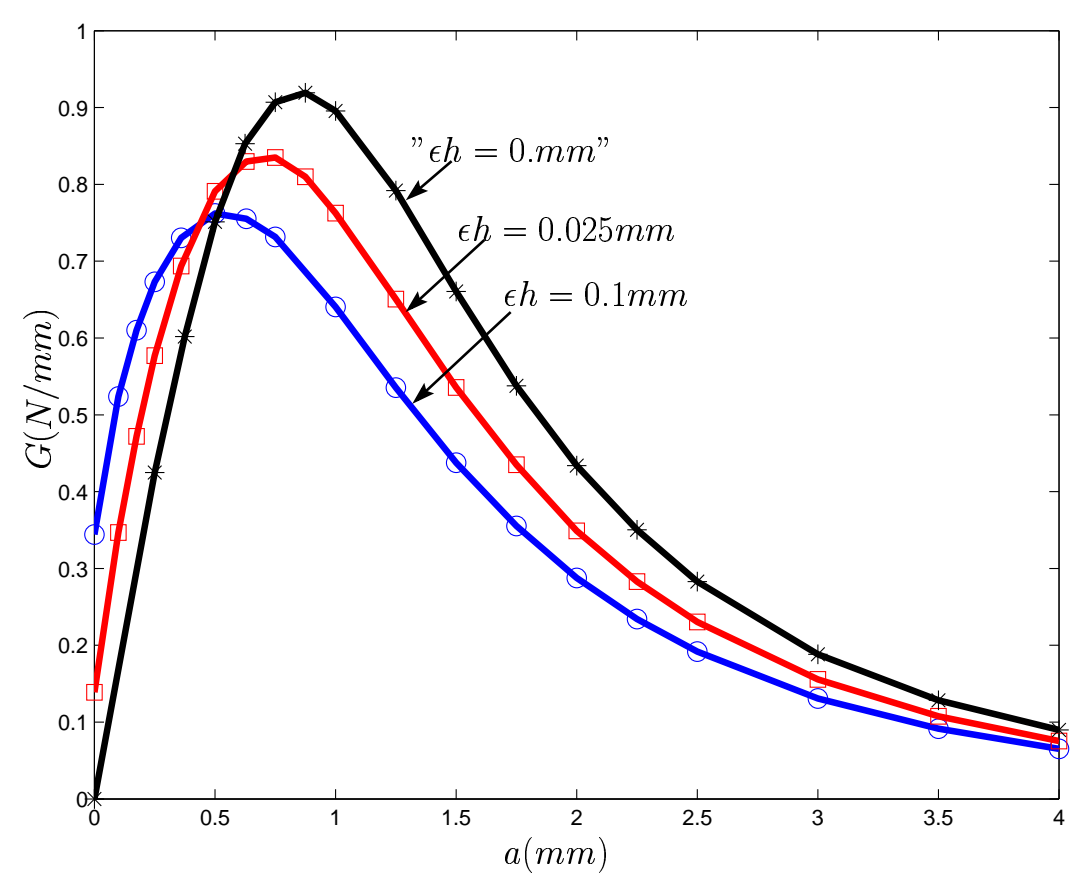

Figure 16: Local energy release rate $G\left(L-a, \frac{l}{2}\right)$ vs. $a$.

\subsection{Circular assembly}

A circular bonded assembly of radius $R=120 \mathrm{~mm}$ was then studied. It was made of two plates of thickness $\frac{e_{p}}{2}=1.5 \mathrm{~mm}$ bonded by an adhesive layer of thickness $0.1 \mathrm{~mm}$, having a centered elliptical hole $H=\left\{\boldsymbol{x}=\left(x_{1}, x_{2}, x_{3}\right) \in \mathbb{R}^{3}, \frac{x_{1}^{2}}{a^{2}}+\frac{x_{2}^{2}}{b^{2}}<1\right\}, b \leq a<R$ (fi gure 17).

The assembly was clamped on the outer boundary $\Gamma_{u}=\left\{x_{1}^{2}+x_{2}^{2}=R^{2}, x_{3}= \pm \frac{e_{p}}{2}\right\}$ and submitted to normal loads $\boldsymbol{f}=\left(0,0, f_{3}^{ \pm}\right)$on $\Gamma_{f}^{ \pm}=\left\{\frac{x_{1}^{2}}{a^{2}}+\frac{x_{2}^{2}}{b^{2}}<1, x_{3}= \pm \frac{e_{p}}{2}\right\}$. Due to this boundary conditions, the joint failed on $\partial H \cap S$. Computations were made using the material characteristics reported in table 1. The fi rst set of computations was devoted to the case of a circular hole. The process of the joint failure was similar to the one of the DCB specimen studied in the paragraph 6.2. As the energy release rate was constant along $\partial H \cap S$, when the control displacement $u_{d}$ exceeded the critical value $u_{d c}$, a circular front of radius $r$ was created in an unstable way. Then, the growth was stable and the crack front remained circular. The critical values at initiation obtained with $a=b=13 \mathrm{~mm}$ are reported in the table 3.

Figures 18 and 19 depict the evolution of the loading factor and the radius $r$ in function of the prescribed displacement. 

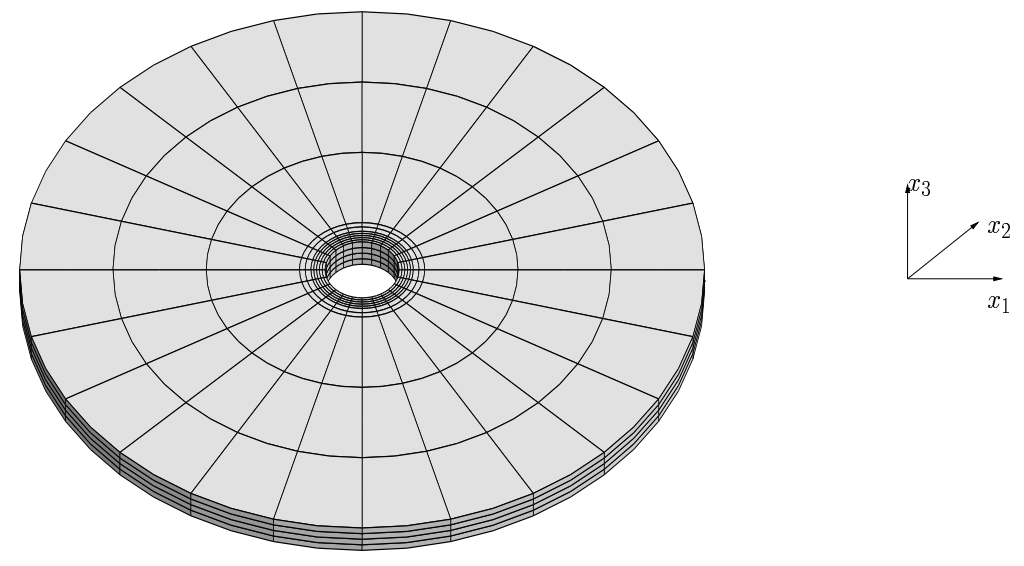

Figure 17: Holed assembly.

\begin{tabular}{cccccc}
\hline$u_{d c}$ & {$\left[u_{3}\right]_{c}$} & $G_{c}$ & $\sigma_{33 c}$ & $\gamma_{33 c}$ & $r$ \\
\hline $0.0106 m m$ & $0.00346 m m$ & $0.397 N / m m$ & $225.975 M P a$ & $3.6 \%$ & $15.691 m m$ \\
\hline
\end{tabular}

Table 3: Critical values on initiation.

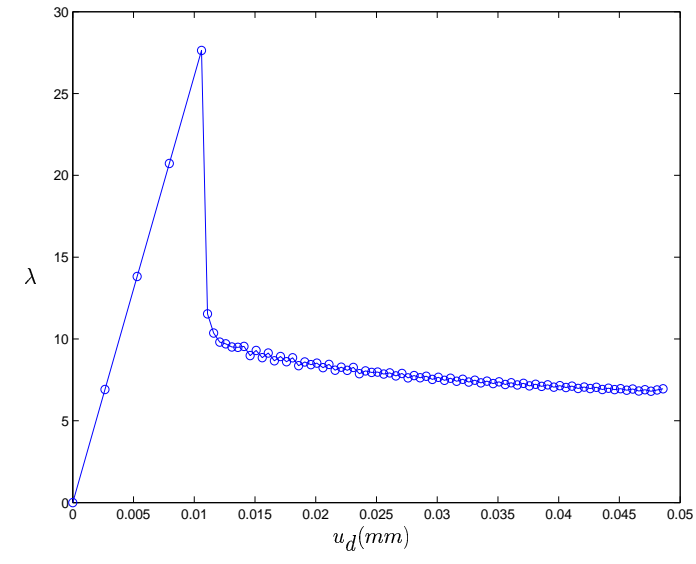

Figure 18: Loading factor $\lambda$ vs. $u_{d}$.

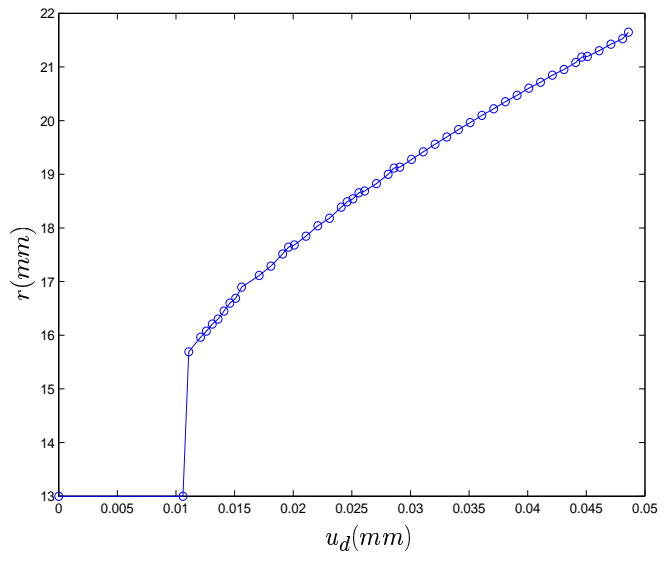

Figure 19: Radius $r$ vs. $u_{d}$. 
In the case of an elliptic hole $(a>b)$, the energy release rate varied along $\partial H \cap S$ as shown in the fi gure 20 where the values along the part $\left\{\left(x_{1}, x_{2}\right)=(\operatorname{acos} \alpha, b \sin \alpha), \alpha \in\left[0, \frac{\pi}{2}\right]\right\}$ of $\partial H \cap S$ are reported. The computations were made taking $a=18 \mathrm{~mm}, b=13 \mathrm{~mm}$ and $u_{d}=0.0101 \mathrm{~mm}$.

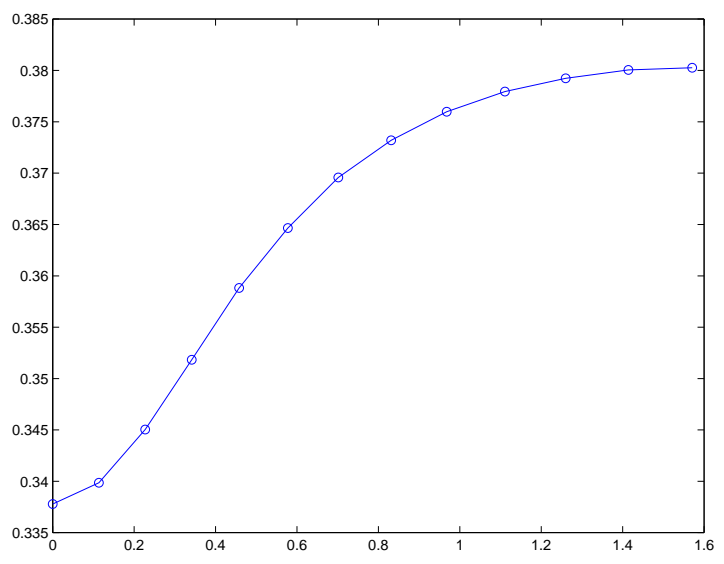

Figure 20: $G(a \cos \alpha, b \sin \alpha)$ vs. $\alpha$.

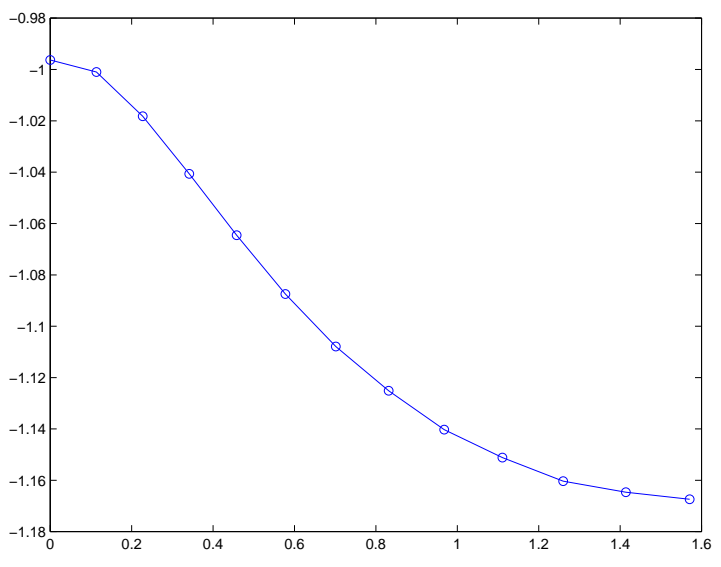

Figure 21: $-\left.\frac{\partial G}{\partial x}\right|_{\partial H} \cdot \vec{n}$ vs. $\alpha$.

The critical energy release rate was fi rstly reached on the point $(0, \pm b)$ corresponding to $\alpha= \pm \frac{\pi}{2}$. However, the unstable character of the growth at initiation ensured that the bond failed on the whole boundary of the hole. With such a choice of loading, the elliptic shape of the front increased the instability. As it can be seen in the fi gure 21, the second derivative of the energy $E$ was negative along $\partial H \cap S$ and reached its minimum for $\alpha= \pm \frac{\pi}{2}$ where the energy release rate was maximum. As a consequence, the normal crack length at initiation was maximal at these points. The different crack positions for several values of the prescribed displacement are reported in the fi gure 22 that shows that the crack front remained elliptic during the growth, with a decreasing ratio $\frac{a}{b}$ up to a value of 1.06 approximately.

The variations of the loading factor and the distance of the front to the hole boundary with respect to the prescribed displacement $u_{d}$ are reported in the fi gures 23 and 24 respectively. 


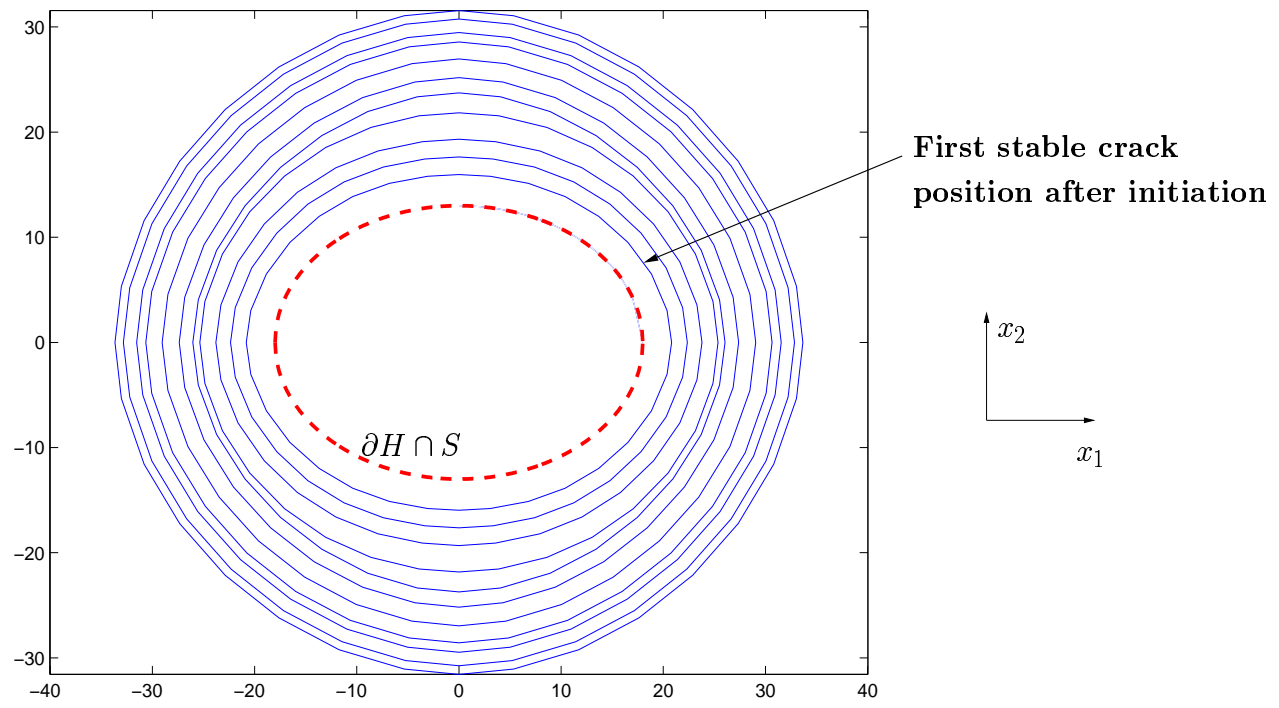

Figure 22: Front positions for $u_{d}=0.0101,0.0131,0.0191,0.0251,0.0371,0.0431$, $0.0551,0.0701,0.0911,0.1031,0.1211(\mathrm{~mm})$.

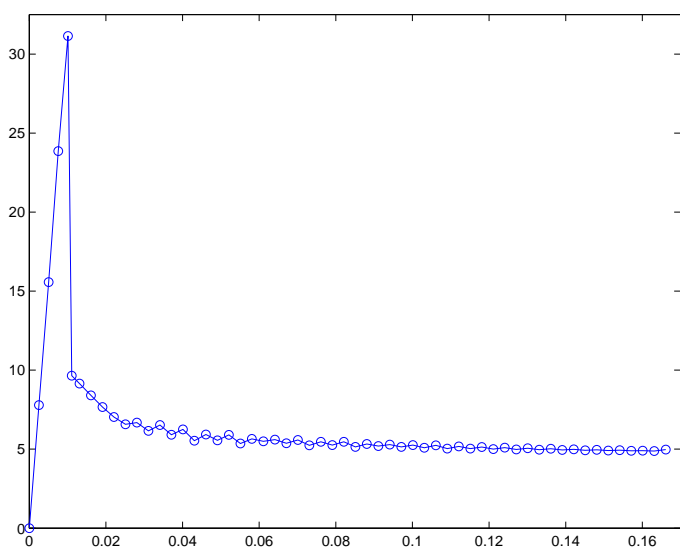

Figure 23: $\lambda$ vs. $u_{d}$.

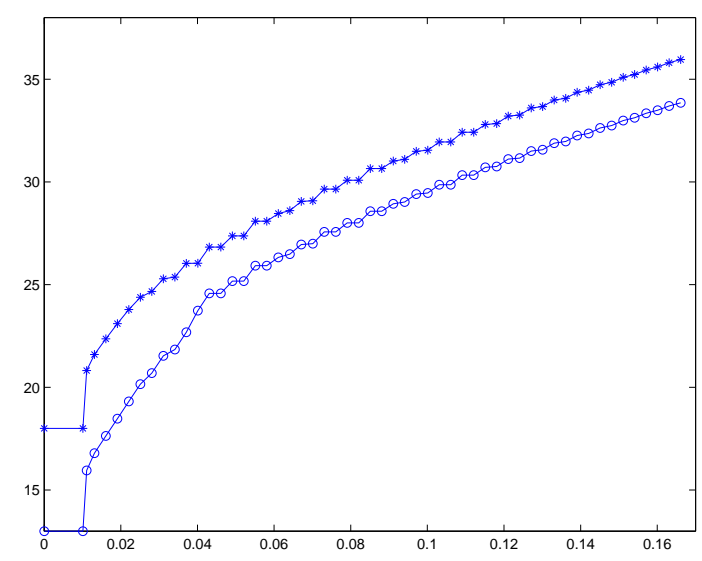

Figure 24: Distance to $\partial H$ vs. $u_{d}$ : ०:point $\alpha=$ $0 ; *$ :point $\alpha=\frac{\pi}{2}$. 


\section{Conclusion}

We have presented an elastic interface model to study adhesively bonded assemblies performing large displacements. It is obtained by an asymptotic expansion technique making the assumption that both the Young's modulus ratio and the thickness ratio of the adhesive and of the adherents are small parameters of the same order. The main characteristic of the model is that the adhesive only has a non linear behaviour expressed in terms of the displacements jumps. It differs therefore form the model proposed by Edlund and Klarbring (Edlund and Klarbring (1992)) where the strains of the mid-surface of the adhesive are taken into account. In addition, it is proved, in the case of small displacements, that the model converges to the perfectly bonded interface model as the adhesive thickness only tends to zero.

A debonding model based on a fracture mechanics approach is then proposed. It consists in minimizing the total energy of the assembly with respect to any admissible crack front displacement. The numerical applications have shown the robustness of the proposed algorithm. In addition, it has allowed to show the unstable character of the debonding growth at initiation on a small length. The simulation of the initiation was presented with the use of the explicit expression of the local energy release rate. In the absence of joint $(\epsilon h=0)$, this expression is equal to zero and can not be used to simulate the initiation of the crack between the two adherents. However, following the approach developed in (Francfort and Marigo (1998)) and writing the conservation of the total energy between the two equilibrium states, just before and after initiation, it is possible to determine simultaneously

the critical load and the fi rst position of the crack front. A numerical simulation is given in (Münch (2002)).

Besides, as the adhesive is assumed much less stiff than the adherents, the small strain's assumption can be questionable. In fact, the elastic interface model and the debonding model associated can be extended to the large strain's case using more general densities of energy than the St-Venant's one. This work is detailed in (Krasucki et al. (2002)).

\section{Appendix A. Spectrum of the operator $D^{(2)}$}

The purpose of this appendix is to show that the spectrum of the operator $D^{(2)}$ admits real positive elements. Using the Green formula, we first write

$$
D^{(2)}(\theta, \theta)=-G_{c} \int_{S_{c_{f}}} \operatorname{det}(\boldsymbol{\nabla} \boldsymbol{\theta}) d S=\frac{1}{2} \int_{c_{f}} G_{c}(\operatorname{div} \boldsymbol{\theta} \boldsymbol{\theta} . \boldsymbol{\nu}-\boldsymbol{\nu} \cdot \boldsymbol{\nabla} \boldsymbol{\theta} \cdot \boldsymbol{\theta}) d \gamma
$$

Let us note $s \in[0, l]$ the curvilinear abscissa along the front $c_{f}$ and $\rho$ the transverse local coordinate to the front. The vector $\boldsymbol{\theta}$ is expressed along the front as follows :

$$
\boldsymbol{\theta}=\theta_{1} \boldsymbol{e}^{\mathbf{1}}+\theta_{2} \boldsymbol{e}^{\mathbf{2}}=\theta_{\tau} \boldsymbol{\tau}+\theta_{\nu} \boldsymbol{\nu}
$$

Then, thanks to the relation $\left.\frac{\partial k}{\partial x}\right|_{\hat{\gamma}_{f}}=k_{, s} \boldsymbol{\tau}+k_{, \rho} \boldsymbol{\nu}$ and the relation

$$
\operatorname{div} \boldsymbol{\theta} \boldsymbol{\theta} . \boldsymbol{\nu}-\boldsymbol{\nu} . \nabla \boldsymbol{\theta} . \boldsymbol{\theta}=\frac{\partial \theta_{1}}{\partial s} \theta_{2}-\frac{\partial \theta_{2}}{\partial s} \theta_{1}
$$


we write

$$
D^{(2)}(\boldsymbol{\theta}, \boldsymbol{\theta})=\frac{1}{2} G_{c} \int_{c_{f}}\left(\frac{\partial \theta_{1}}{\partial s} \theta_{2}-\frac{\partial \theta_{2}}{\partial s} \theta_{1}\right) d \gamma
$$

Then, since $D^{(2)}$ is a symmetric bilinear form, the use of the relation :

$$
D^{(2)}(\boldsymbol{\theta}, \boldsymbol{\psi})=\frac{1}{4}\left(D^{(2)}(\boldsymbol{\theta}+\boldsymbol{\psi}, \boldsymbol{\theta}+\boldsymbol{\psi})-D^{(2)}(\boldsymbol{\theta}-\boldsymbol{\psi}, \boldsymbol{\theta}-\boldsymbol{\psi})\right)
$$

gives :

$$
D^{(2)}(\boldsymbol{\theta}, \boldsymbol{\psi})=\frac{1}{2} G_{c} \int_{c_{f}}\left(\frac{\partial \theta_{1}}{\partial s} \psi_{2}-\frac{\partial \theta_{2}}{\partial s} \psi_{1}\right) d \gamma
$$

Then, the elements $\lambda$ of the spectrum characterized by the relation :

$$
D^{(2)}(\boldsymbol{\theta}, \boldsymbol{\psi})=\lambda(\boldsymbol{\theta}, \boldsymbol{\psi}) \equiv \lambda \int_{c_{f}} \boldsymbol{\theta} . \boldsymbol{\psi} d \gamma
$$

are solution of the following parabolic differential system $\left(\Lambda=2 G_{c}^{-1} \lambda\right)$ :

$$
\left(\begin{array}{cc}
0 & -1 \\
1 & 0
\end{array}\right)\left(\begin{array}{l}
\frac{\partial \theta_{1}}{\partial s} \\
\frac{\partial \theta_{2}}{\partial s}
\end{array}\right)+\left(\begin{array}{cc}
-\Lambda & 0 \\
0 & -\Lambda
\end{array}\right)\left(\begin{array}{l}
\theta_{1} \\
\theta_{2}
\end{array}\right)=\left(\begin{array}{l}
0 \\
0
\end{array}\right) .
$$

The characteristic polynomial $p$ is obtained with the choice $\boldsymbol{\theta}=e^{\beta s} \tilde{\boldsymbol{\theta}}, \beta \in \mathbb{C}$. Let us remark that the condition $\boldsymbol{\theta}(s=0,0)=\boldsymbol{\theta}(s=l, 0)$ implies $\beta=i \omega ; \omega=\frac{2 n \pi}{l} \in \mathbb{R}$. This implies :

$$
\left(\begin{array}{cc}
-\Lambda & -\beta \\
\beta & -\Lambda
\end{array}\right)\left(\begin{array}{c}
\tilde{\theta}_{1} e^{\beta s} \\
\tilde{\theta}_{2} e^{\beta s}
\end{array}\right)=\left(\begin{array}{l}
0 \\
0
\end{array}\right)
$$

and finally :

$$
p(\Lambda)=\Lambda^{2}+\beta^{2}=\Lambda^{2}-\omega^{2}=0 .
$$

The eigenvalues of $D^{(2)}$ are :

$$
\lambda_{n}= \pm G_{c} \frac{\pi n}{l} \quad n \in \mathbb{N}
$$

and we conclude that the spectrum is symmetrical with respect to zero. The sets $\left\{\theta ; D^{(2)}(\theta, \theta)\right\}$ and $V_{\theta}$ defined in (27) are not empty.

\section{Appendix B. Expression of the energy release rate as a curvilinear integral}

The purpose of this appendix is to show how we express the energy release rate $g$ defined on the vicinity $S_{\theta}$ of $c_{f}$ as a curvilinear integral along $c_{f}$. The starting point is the expression (21). We decompose $\Omega_{\epsilon}^{ \pm}$as 
follow $\Omega_{\epsilon}^{ \pm}=B_{R} \cup \Omega_{R} ; B_{R}$ design a cylinder of radius $R$ centered on the crack front $c_{f} \subset S$. Let us note $S_{\Omega_{R}}=S \cap \Omega_{R}, c_{R}=S \cap \partial \Omega_{R}$ and $\bar{g}(\boldsymbol{\theta})$ the part of $g(\boldsymbol{\theta})$ restricted to $B_{R}$ such that :

$$
\begin{aligned}
g(\boldsymbol{\theta})= & \bar{g}(\boldsymbol{\theta})+\int_{\Omega_{R}} \operatorname{Tr}(\boldsymbol{\sigma} . \nabla \boldsymbol{u} . \nabla \boldsymbol{\theta}) d \Omega-\frac{1}{2} \int_{\Omega_{R}} \operatorname{Tr}(\boldsymbol{\sigma} . \nabla \boldsymbol{u}) \operatorname{div} \boldsymbol{\theta} d \Omega \\
& -\frac{1}{2} \int_{S_{\Omega_{R}}}\left[\sigma_{\alpha 3}^{m}\left[u_{\alpha}\right]+\sigma_{33}^{m}\left(\left[u_{3}\right]+\frac{1}{2 \epsilon h}\left[u_{k}\right]\left[u_{k}\right]\right)\right] \operatorname{div} \boldsymbol{\theta} d S .
\end{aligned}
$$

Using the green formula and the fact that the normal vector to $S$ is directed by $\boldsymbol{e}_{\mathbf{3}}$, the first term on $\Omega_{R}$ becomes :

$$
\int_{\Omega_{R}} \operatorname{Tr}(\boldsymbol{\sigma} \cdot \nabla \boldsymbol{u} \cdot \nabla \boldsymbol{\theta}) d \Omega=-\int_{\Omega_{R}}\left(\sigma_{i j} u_{j, k} \theta_{k}\right)_{, i} d \Omega-\int_{\partial \Omega_{R}} \sigma_{i j} u_{j, k} \theta_{k} \nu_{i} d \Gamma-\int_{S_{\Omega_{R}}}\left[\sigma_{3 j} u_{j, k}\right] \theta_{k} d S
$$

where $\boldsymbol{\nu}$ is the outer normal to $B_{R}$. Let us note that the jump term [.] is not present in the two others terms of $g(\boldsymbol{\theta})-\bar{g}(\boldsymbol{\theta})$, due to the relation $\boldsymbol{\theta} . \boldsymbol{\nu}=\theta_{3} e_{3} \equiv 0$ on $S$ : we obtain

$$
\begin{aligned}
g(\boldsymbol{\theta})= & \bar{g}(\boldsymbol{\theta})+\frac{1}{2} \int_{\Omega_{R}}\left(\sigma_{i j} u_{j, i}\right)_{, k} \theta_{k} d \Omega-\int_{\Omega_{R}}\left(\sigma_{i j} u_{j, k}\right)_{i} \theta_{k} d \Omega+\frac{1}{2} \int_{\partial \Omega_{R}} \operatorname{Tr}(\boldsymbol{\sigma} . \nabla \boldsymbol{u}) \boldsymbol{\theta} . \boldsymbol{\nu} d \Gamma \\
& -\int_{\partial \Omega_{R}} \sigma_{i j} u_{j, k} \theta_{k} \nu_{i} d \Gamma+\frac{1}{2} \int_{S_{\Omega_{R}}}\left[\sigma_{\alpha 3}\left[u_{\alpha}\right]+\sigma_{33}\left(\left[u_{3}\right]+\frac{1}{2 \epsilon h}\left[u_{l}\right]\left[u_{l}\right]\right)\right]_{, k} \theta_{k} d S \\
& -\int_{S_{\Omega_{R}}}\left[\sigma_{3 j} u_{j, k}\right] \theta_{k} d S+\frac{1}{2} \int_{c_{R}}\left[\sigma_{\alpha 3}\left[u_{\alpha}\right]+\sigma_{33}\left(\left[u_{3}\right]+\frac{1}{2 \epsilon h}\left[u_{l}\right]\left[u_{l}\right]\right)\right] \boldsymbol{\theta} . \boldsymbol{\nu} d \gamma .
\end{aligned}
$$

Terms on $\Omega_{R}$ vanish. Indeed, they are equal to $\frac{1}{2}\left(\sigma_{i j} u_{i, j}\right)_{, k}-\left(\sigma_{i j} u_{j, k}\right)_{, i}$ then, according to $\sigma_{i j, j}=0$, they are also equal to $\frac{1}{2} \sigma_{i j, k} u_{j, i}-\frac{1}{2} \sigma_{i j} u_{u, i j}$. Using the symmetries of the stiffness tensor $\mathbb{R}$ and $\sigma_{i j}=\frac{1}{2} R_{i j \tau \theta}\left(u_{\tau, \theta}+u_{\theta, \tau}\right)$ in the adherents, the remaining term is equal to zero. In a similarly way, the terms on $S_{\Omega_{R}}$ vanish. Indeed, the stresses $\sigma_{3 j}$ are constant in the joint and we have $\left[\sigma_{3 j} u_{j, k}\right]=\sigma_{3 j}\left[u_{j, k}\right]$. Then, according to the relations of transmission (8), it comes :

$$
\sigma_{3 j}\left[u_{j, k}\right]=\sigma_{3 j}\left[u_{j}\right]_{, k}=\sigma_{3 j}^{m}\left[u_{j}\right]_{, k}+\sigma_{33}^{m} \frac{\left[u_{j}\right]}{\epsilon h}\left[u_{j}\right]_{, k}
$$

whereas the symmetry of the relation (7) in the joint leads to :

$$
\frac{1}{2}\left[\sigma_{\alpha 3}^{m}\left[u_{\alpha}\right]+\sigma_{33}^{m}\left(\left[u_{3}\right]+\frac{1}{2 \epsilon h}\left[u_{l}\right]\left[u_{l}\right]\right)\right]_{, k}=\sigma_{\alpha 3}^{m}\left[u_{\alpha}\right]_{, k}+\sigma_{33}^{m}\left(\left[u_{3}\right]_{, k}+\frac{\left[u_{l}\right]}{\epsilon h}\left[u_{l}\right]_{, k}\right),
$$

then to :

$$
\begin{aligned}
g(\boldsymbol{\theta})= & \bar{g}(\boldsymbol{\theta})+\frac{1}{2} \int_{\partial \Omega_{R}} \operatorname{Tr}(\boldsymbol{\sigma} \cdot \nabla \boldsymbol{u}) \boldsymbol{\theta} \cdot \boldsymbol{\nu} d \Gamma-\int_{\partial \Omega_{R}} \sigma_{i j} u_{j, k} \theta_{k} \nu_{i} d \Gamma \\
& +\frac{1}{2} \int_{c_{R}}\left[\sigma_{\alpha 3}^{m}\left[u_{\alpha}\right]+\sigma_{33}^{m}\left(\left[u_{3}\right]+\frac{1}{2 \epsilon h}\left[u_{l}\right]\left[u_{l}\right]\right)\right] \boldsymbol{\theta} . \boldsymbol{\nu} d \gamma
\end{aligned}
$$

Finally, $g$ being independent of $R$, the former expression remains true for $R$ arbitrarily small. If the adherents are convex, the study of the stress's singularity, in the case of weak interface, permits to conclude, in a similarly way as for the linear case (Destuynder et al. (1992)), that $\bar{g}(\boldsymbol{\theta})$ and integrals on $\partial \Omega_{R}$ converge toward zero with $R$. Finally, we obtain the expression (22) which shows that the energy release is independent of the vector $\boldsymbol{\theta}$ in a vicinity of $c_{f}$. 


\section{References}

Ait Moussa, A., 1996. Comportement asymptotique d'une bande élastique mince. Les Annales Maghrébines de l’Ingénieur, Vol. 10 (2).

Alfano, G., Crisfield, M.A., 2001. Finite element interface models for the delamination analysis of laminated composites: mechanical and computational issues. Int. J. Numer. Meth. Eng. 50, pp. 1701-1736.

Allix, O., Corigliano, A., 1999. Geometrical and interfacial non-linearities in the analysis of delamination in composites. Int J. Solids Structures. 36, pp. 2189-2216.

Bruno, D., Grimaldi, A., 1990. Delamination failure of layered composite plates loaded in compression. Int J. Solids Structures Vol. 26 (3), pp. 313-330.

Chaboche, J.L., Feyel, F., Monerie, Y., 2001. Interface debonding models: a viscous regularization with a limited rate dependency. Int. J. Solids. Struct. 38, pp. 3127-3160.

Chai, H., 1986. On the correlation between the mode I failure of the adhesive joints and laminated composites, Eng. Fract. Mechs. Vol.24 3 413-431.

Ciarlet, P.G., Destuynder. P., 1979. A justification of a nonlinear model in plate theory, Comput. Methods. Appl. Mech. Engrg. 17/18, pp.227-258.

Ciarlet, P.G., 1988. Mathematical elasticity, Vol I, North-Holland.

Delale, F., Erdogan, F., Aydinoglu, M.N., 1981. Stresses in adhesively bonded joints: a closed form solution. J. of Comp. Mat. 15, pp. 249-271.

Destuynder, P., Djaoua, M., 1981. Sur une interprétation mathématique de l'intégrale de Rice en théorie de la rupture fragile. Math. Meth. Appl. Sci. 3, pp. 179-207.

Destuynder, P., Michavila, F., Santos, A., Ousset, Y., 1992. Some theoretical aspects in computational analysis of adhesive lap joints. Int. J. Numer. Methods. Eng., 35, pp. 1237-1262.

Destuynder, P., Ousset, Y., Stackler, C., 1988. Sur les singularités de contraintes dans les joints collés. J. Mec. Theor. Appli., Vol. 7(6), pp. 899-926.

Edlund, U., Klarbring, A., 1992. A geometrically nonlinear model of the adhesive joint problem and its numerical treatment, Comput. Methods Appl. Mech. Engrg., pp. 327-350.

Edlund, U., 1994. Surface adhesive joint description with coupled elastic-plastic damage behaviour and numerical applications. Comput. Methods Appl Mech Engrg, 115, pp. 253-276.

Francfort, G.A., Marigo, J.J., 1998. Revisiting brittle fracture as an energy minimisation problem. J. Mech. Phys. Solids. 46(8), pp. 1319-1342.

Geymonat, G., Krasucki, F., Lenci, S., 1999. Mathematical analysis of a bonded joint with a soft thin adhesive. Mathematics and Mechanics of Solids, 4, pp. 201-255. 
Geymonat, G., Krasucki, F., Marini, D., Vidrascu, M., 1998. A domain decomposition method for a bonded structure. Mathematical Models and Methods in Applied Sciences, Vol.8, pp. 1387-1402.

Goland, M., Reissner, E., 1944. The stresses in cemented joints. J. Appl. Mech., ASME, 11, A17-A27.

Harris, J.A., Adams, R.D, 1984. Stenght prediction of bonded simple lap joints by nonlinear finite element methods. Int J. Adhesion and adhesives, Vol. 4 (2), pp. 65-78.

Herskovits, J., 1992. An interior point technique for nonlinear optimization. RR INRIA N 1808 .

Klarbring, A., 1991. Derivation of a model of adhesively bonded joints by the asymptotic expansion method. Int. J. Eng Sci. 29 pp. 493-512.

Krasucki F., Münch, A., Ousset, Y., 2001. Asymptotic analysis of a bonded joint in nonlinear elasticity. C.R.Acad Sci. Paris, Serie IIb, t. 329. Vol. 6, pp. 429-434.

Krasucki F., Münch, A., Ousset, Y., 2002. Mathematical analysis of nonlinear bonded joint models, To appear in Mathematics and Mechanics of Solids.

Ladevèze, P., 1992. A damage computational method for composite structures. Comput. Struct. 22, pp. 79-87.

Leguillon, D., 2002. Strength or Toughness ? A criterion for crack onset at a notch. Eur. J. Mech. A/Solids 21, pp. 61-72.

Lions, J-L., 1973. Pertubations singulières dans les problèmes aux limites et en controle optimal. Springer Verlag, Berling.

Möes, N., Dolbow, J., Belytschko, T., 1999. A finite element method for crack growth without remeshing. Int. J. Numer. Meth. Eng., 46(1), pp. 131-150.

Münch, A., Ousset, Y., 2002. Numerical simulation of delamination growth in curved interfaces. Comput. Methods Appl. Mech. Engrg. 191, pp. 2045-2067.

Münch, A., 2002. Propagation de fissures tridimensionnelles dans les structures stratifiées : Interfaces courbes et assemblages collés, PhD. Thesis, Paris VI.

Nguyen, Q.S., 2000. Stability and Nonlinear Solids Mechanics. John Wiley and Sons, Chichester.

Ousset, Y., 1999. Numerical simulation of delamination growth in layered composites plates. Eur. J. Mech. A/Solids 18, pp. 291-312.

Reddy, J.N., Roy, S., 1988. Nonlinear analysis of adhesively bonded joints. Int J. Nonlinear Mechanics, Vol. 23, No.2, pp. 97-112.

Robinson, P., Song, D.Q., 1992. A modified DCB specimen for mode I testing of multidirectional laminates. J. Compos. Materials, Vol. 26 (11), pp.1554-1577.

Rose, L.R.F., 1987. Crack reinforcement by distributed springs. J. Mech. Phys. Solids, 34 pp. 383-405. 
Roudolff, F., Ousset, Y., 2002. Comparison between two approaches for the simulation of delamination growth in a DCB specimen. Aerosp. Sci. and Technol. Vol 2, pp. 123-130.

Tvergaard, V., 1990. Effect of fibre debonding in a whisker-reinforced metal. Mater. Sci. Engng. A 125, pp. 203-213.

Wells, G.N., Sluys, L.J., 2000. A new method for modelling cohesive cracks using finite elements. Int. J. Numer. Meth. Eng., 50, pp. 2667-2682.

Wooley, G.R., Carver, D.R., 1971. Stress concentration factors for bonded lap joints. J. Aircraft 8 (10), pp. 817-820. 